\title{
DEVELOPMENT OF CMPS METHOD FOR ACCURATE WATER-SURFACE TRACKING IN BREAKING WAVES
}

$\operatorname{AUTHOR}(S):$

KHAYYER, ABBAS; GOTOH, HITOSHI

CITATION:

KHAYYER, ABBAS ... [et al]. DEVELOPMENT OF CMPS METHOD FOR ACCURATE WATERSURFACE TRACKING IN BREAKING WAVES. Coastal Engineering Journal 2008, 50(2): 179207

\section{ISSUE DATE:}

2008

URL:

http://hdl.handle.net/2433/93447

\section{RIGHT:}

Electronic version of an article published as Coastal Engineering Journal, 50, 2, 2008, 179-207. DOI:

10.1142/S0578563408001788 @ copyright World Scientific Publishing Company http://www.worldscinet.com/cej/; This is not the published version. Please cite only the published version.; この論文は出版社版でありません。引用の際には 出版社版をご確認ご利用ください。 


\title{
DEVELOPMENT OF CMPS METHOD FOR ACCURATE WATER-SURFACE TRACKING IN BREAKING WAVES
}

\author{
ABBAS KHAYYER \\ Department of Urban and Environmental Engineering, \\ Kyoto University, Katsura Campus, Nishikyo-ku, \\ Kyoto 615-8540, Japan \\ khayyer@wfee.mbox.media.kyoto-u.ac.jp \\ HITOSHI GOTOH* \\ Department of Urban and Environmental Engineering, \\ Kyoto University, Katsura Campus, Nishikyo-ku, \\ Kyoto 615-8540, Japan \\ gotoh@mbox.kudpc.kyoto-u.ac.jp
}

Received

Revised 
A Corrected Moving Particle Semi-implicit (CMPS) method is proposed for accurate tracking of water surface in breaking waves. The original formulations of standard MPS method are revisited from the view point of momentum conservation. Modifications and corrections are made to ensure the momentum conservation in a particle-based calculation of viscous incompressible free-surface flows. A simple numerical test demonstrates the excellent performance of the CMPS method in exact conservation of linear momentum and significantly enhanced preservation of angular momentum. The CMPS method is applied to the simulation of plunging breaking and post-breaking of solitary waves. Qualitative and quantitative comparisons with the experimental data confirm the high capability and precision of the CMPS method. A tensor-type strain-based viscosity is also proposed for further enhanced CMPS reproduction of a splash-up.

Keywords: MPS method; CMPS method; momentum conservation; breaking waves; splash-up; strain-based viscosity. 


\section{Introduction}

Free surface flows are of crucial importance in the field of hydraulic engineering but are difficult to simulate since the surface boundary conditions are specified on an arbitrary moving surface. Numerous grid-based water-surface-tracking techniques, such as the MAC [Harlow and Welch, 1965] or VOF [Hirt and Nichols, 1981] methods were proposed to tackle the difficulty in free surface modeling. Nevertheless, both MAC and VOF methods suffer from the problem of numerical diffusion arising from the grid-based discretization of advection terms in the Navier-Stokes equation. The numerical diffusion becomes significant when the free surface experiences abrupt and large deformations accompanied by fluid fragmentation (such as the case of a plunging breaking wave and resultant splash-up). A few algorithms such as the CIP method [Yabe et al., 1991; Yabe et al., 2001] has been proposed to attenuate the numerical diffusion; yet, the implementation of such sophisticated algorithms would further complicate the computational procedure for free surface modeling.

A recent interest has been focused on the development of the next generation computational methods, the gridless or meshfree methods that are expected to be superior to the conventional grid-based methods. Meshfree methods provide substantial potential in many classes of problems, such as those which include moving discontinuities and those characterized by large deformations. The main idea behind the meshfree methods is to obtain accurate and stable numerical solutions for integral equations of partial differential equations with all kinds of possible boundary conditions with a set of arbitrarily distributed calculation points such as nodes or particles. The calculation points can be either fixed (Eulerian approach) or moveable with the fluid (Lagrangian approach). The Lagrangian meshfree method is called the particle method. Particle methods can be classified into those based on field approximations, as the Element-Free Galerkin method (EFG), and those based on kernel approximations, as the Smoothed Particle Hydrodynamics (SPH) or Moving Particle Semi-implicit (MPS) method. 
Originally developed by Koshizuka et al. [1995], the MPS method has been applied in a wide variety of problems such as elastic structures [Koshizuka et al., 2001a], nuclear reactor safety [Koshizuka et al. 2001b] or blood flow simulation [Tsubota et al., 2006]. The MPS method has been improved and extended into coastal engineering to study wave breaking [Gotoh and Sakai, 1999; Gotoh et al., 2005] and two-phase sediment-water interactions [Gotoh et al., 2001a]. Despite being a superior method for the calculation of hydraulic phenomena, there are some shortcomings associated with the MPS method. Being similar to some other particle methods, such as the SPH method, the MPS method suffers from some inherent difficulties such as non-conservation of mechanical properties (namely, mechanical energy or momentum) or lack of completeness of kernel interpolants. In case of the SPH method, a huge amount of research has been carried out leading to Corrective SPH (CSPH) methods such as those which ensure the preservation of linear and angular momentum [e.g. Bonet and Lok, 1999] or those which guarantee the high-order completeness of interpolants [e.g. Liu et al., 1995]. In case of the MPS method, however, there have been much less studies regarding to the mentioned difficulties. By treating the continuum as a Hamiltonian system of particles and through a direct discretization of the Lagrangian for inviscid incompressible flows, Suzuki et al. [2007] have developed the HMPS (Hamiltonian MPS) in which the momentum and mechanical energy of the system are preserved. Although HMPS is superior to the original or standard MPS in terms of the preservation of conservation laws, the numerical framework of HMPS is much more complicated than that of the standard MPS. Hence, it would be more difficult to code and implement a HMPS calculation than a standard MPS one.

This paper is focused on the momentum conservation properties of the standard MPS formulations. The aim is to improve the performance of the standard MPS method by modifying and correcting the formulations while maintaining their robustness and simplicity. The target phenomenon is a strong plunging breaking and resultant splash-up, where momentum conservation properties of formulations become more important. In addition, as the splash-up is a highly 
deformed flow characterized by non-uniform strain rates, it would be preferable to obtain the viscous forces by applying a tensor-type strain-based viscosity. In this paper, we propose a tensor-type strain-based viscosity when the Corrected MPS (CMPS) method is supposed to simulate a highly non-uniformly deformed flow such as the splash-up.

The paper is organized in the following way. Firstly, a brief explanation of the MPS method including the governing equations and the equation solvers is presented. Next, the momentum conservation properties of standard MPS formulations are discussed. Following by that, the new Corrected MPS (CMPS) formulations are derived with the aim of ensuring the conservation of linear and angular momentum in a MPS-based calculation of viscous incompressible flow. After a simple verification, the proposed CMPS method is applied to the simulation of solitary wave breaking and post-breaking on a plane slope. The enhanced performance of the proposed CMPS method is proved through both qualitative and quantitative comparisons with the experimental data.

\section{MPS Method}

In this section the MPS method is briefly explained. Detailed descriptions were provided by Koshizuka and Oka [1996] or Gotoh et al. [2005]. The MPS method is a macroscopic, deterministic particle method proposed by Koshizuka et al. [1995]. The fluid is modeled as an assembly of interacting particles, the motion of which is calculated through the interactions with neighboring particles and according to the governing equation of fluid motion. The governing equations are the continuity equation and Navier-Stokes equation describing the motion of a viscous incompressible flow:

$$
\begin{gathered}
\nabla \cdot \boldsymbol{u}=0 \\
\frac{\mathrm{D} \boldsymbol{u}}{\mathrm{D} t}=-\frac{1}{\rho} \nabla p+\boldsymbol{g}+\nu \nabla^{2} \boldsymbol{u}
\end{gathered}
$$

where $\boldsymbol{u}=$ particle velocity vector; $t=$ time; $\rho=$ fluid density; $p=$ particle pressure; $\boldsymbol{g}=$ gravitational 
acceleration vector and $v=$ laminar kinematic viscosity. The left hand side of Eq. 2 denotes the Lagrangian derivative involving the advection term. In the particle methods, including the MPS method, the advection term is automatically calculated through the tracking of particle motion; hence, the numerical diffusion arising from the successive interpolation of the advection function in Eulerian grid-based methods is controlled without using a sophisticated algorithm. The above equations are discretized by use of particle interaction models, namely the gradient and Laplacian operators.

The gradient operator is a local weighted average of the gradient vectors between particle $i$ and its neighboring particles $j$ :

$$
\langle\nabla \phi\rangle_{i}=\frac{D_{s}}{n_{0}} \sum_{j \neq i} \frac{\phi_{j}-\phi_{i}}{\left|\boldsymbol{r}_{j}-\boldsymbol{r}_{i}\right|^{2}}\left(\boldsymbol{r}_{j}-\boldsymbol{r}_{i}\right) w\left(\left|\boldsymbol{r}_{j}-\boldsymbol{r}_{i}\right|\right)
$$

where $\phi=$ arbitrary scalar function, $D_{s}=$ number of space dimensions, $\boldsymbol{r}=$ coordinate vector of fluid particle, $w(r)=$ the kernel function and $n_{0}=$ the constant particle number density. Following Koshizuka et al. [1998], the pressure gradient is defined by replacing $\phi_{i}$ in Eq. 3 by the minimum value of $\phi$ among the neighboring particles, such as:

$$
\begin{array}{r}
\langle\nabla p\rangle_{i}=\frac{D_{s}}{n_{0}} \sum_{j \neq i} \frac{p_{j}-\hat{p}_{i}}{\left|\boldsymbol{r}_{j}-\boldsymbol{r}_{i}\right|^{2}}\left(\boldsymbol{r}_{j}-\boldsymbol{r}_{i}\right) w\left(\left|\boldsymbol{r}_{j}-\boldsymbol{r}_{i}\right|\right) \\
\hat{p}_{i}=\min _{j \in J}\left(p_{i}, p_{j}\right) \quad, J=\left\{j: w\left(\left|\boldsymbol{r}_{j}-\boldsymbol{r}_{i}\right|\right) \neq 0\right\}
\end{array}
$$

This replacement improves the stability of the code by ensuring the interparticle repulsive force [Koshizuka et al., 1998]. The most common kernel function applied in MPS research is the one proposed by Koshizuka and Oka [1996]:

$$
w(r)= \begin{cases}\frac{r_{e}}{r}-1 & 0 \leq r<r_{e} \\ 0 & r_{e} \leq r\end{cases}
$$

The particle number density at the $i$-th particle position is defined in the following form by using the 
kernel function:

$$
\langle n\rangle_{i}=\sum_{j \neq i} w\left(\left|\boldsymbol{r}_{j}-\boldsymbol{r}_{i}\right|\right)
$$

The Laplacian operator is derived based on the concept of diffusion and has the following form [Koshizuka et al., 1998]:

$$
\left\langle\nabla^{2} \phi\right\rangle_{i}=\frac{2 D_{s}}{n_{0} \lambda} \sum_{j \neq i}\left(\phi_{j}-\phi_{i}\right) w\left(\mid \boldsymbol{r}_{j}-\boldsymbol{r}_{i}\right)
$$

In the above equation the parameter $\lambda$ is introduced to make the increase of variance due to the re-distribution of the above equation equal to the increase of variance estimated from the unsteady diffusion equation. $\lambda$ is introduced as:

$$
\lambda=\frac{\sum_{j \neq i} w\left(\left|\boldsymbol{r}_{j}-\boldsymbol{r}_{i}\right|\right)\left|\boldsymbol{r}_{j}-\boldsymbol{r}_{i}\right|^{2}}{\sum_{j \neq i} w\left(\left(\boldsymbol{r}_{j}-\boldsymbol{r}_{i} \mid\right)\right.}
$$

The iterative prediction-correction process in the MPS method is composed of two main steps. The first prediction step is an explicit integration in time without enforcing incompressibility, while, the second correction step is an implicit computation of a divergence free velocity field. In the first process, intermediate temporal particle velocities and positions are obtained without considering the pressure term. In this process the mass conservation or the incompressibility of fluid is not satisfied, in other words, the number densities $n^{*}$ that are calculated at the end of first process deviate from the constant $n_{0}$; hence, a second corrective process is required to adjust the number densities to initial constant values prior to the time step. In the second process, the intermediate particle velocities are updated through solving the Poisson equation of pressure which contains the deviation of particle number densities. The Poisson equation of pressure is derived as [Koshizuka et al., 1995]:

$$
\left(\nabla^{2} p_{k+1}\right)_{i}=\frac{\rho}{(\Delta t)^{2}} \frac{\left(n_{k}^{*}\right)_{i}-n_{0}}{n_{0}}
$$


where $\Delta t=$ calculation time step; and $k$ denotes the step of calculation. In the MPS method, if the particle number density satisfies the following condition:

$$
n_{i}<\beta n_{0}
$$

the particle is considered as a free-surface particle (constant $\beta=0.97$ ) for which the zero pressure boundary condition is applied [Koshizuka and Oka, 1996].

\section{Momentum Conservation Properties of MPS Formulations}

\subsection{Conservation of linear momentum}

The total linear momentum of a system of particles is given by:

$$
\boldsymbol{G}=\sum_{i=1}^{N} m_{i} \boldsymbol{u}_{i}
$$

where $N=$ total number of fluid particles; $m_{i}$ and $\boldsymbol{u}_{i}$ represent the mass and velocity vector of particle $i$, respectively. The motion of each particle is governed by the Newton's second law:

$$
\boldsymbol{F}_{i}-\boldsymbol{A}_{i}=m_{i} \boldsymbol{a}_{i}
$$

where $\boldsymbol{F}_{i}$ and $\boldsymbol{A}_{i}$ denote the external and internal vector forces acting on particle $i$ and $\boldsymbol{a}_{i}$ is the instantaneous particle acceleration. In the absence of external forces, the rate of time change of total linear momentum $(=\dot{\boldsymbol{G}})$ is:

$$
\dot{\boldsymbol{G}}=\sum_{i=1}^{N} m_{i} \boldsymbol{a}_{i}=-\sum_{i=1}^{N} \boldsymbol{A}_{i}
$$

Thus the condition for preservation of linear momentum can simply be written as:

$$
\sum_{i=1}^{N} \boldsymbol{A}_{i}=0
$$

In general the internal force on particle $i$ can be expressed as the sum of interaction forces between pairs of particles: 


$$
\boldsymbol{A}_{i}=\sum_{j=1}^{N_{i}} \boldsymbol{A}_{i j}
$$

where $N_{i}=$ the total number of neighboring particles of particle $i ; \boldsymbol{A}_{i j}=$ internal interacting vector forces between particle $i$ and its neighboring particle $j$. In Fig. 1, a pair of particles, particle $i$ and its neighboring particle $j$ are shown schematically with the internal interaction forces between them. The internal interaction forces can be decomposed into pressure and viscous interaction forces as depicted in Fig. 2. It can be shown that the linear momentum is exactly conserved for the viscous forces (Fig. 2(b)) because the same magnitude of viscous forces works in the opposite direction; however, the pressure interacting forces do not preserve linear momentum (Fig. 2(a)). Considering Newton's second law and from Eqs. 2 and 4, the force due to pressure on particle $i$ owing to $j$ is:

$$
\boldsymbol{A}_{j \rightarrow i}^{p}=-m_{i} \frac{D_{s}}{\rho n_{0}} \frac{p_{j}-\hat{p}_{i}}{\left|\boldsymbol{r}_{j}-\boldsymbol{r}_{i}\right|^{2}}\left(\boldsymbol{r}_{j}-\boldsymbol{r}_{i}\right) w\left(\left|\boldsymbol{r}_{j}-\boldsymbol{r}_{i}\right|\right)
$$

while the pressure force on particle $j$ owing to $i$ would be:

$$
\boldsymbol{A}_{i \rightarrow j}^{p}=-m_{j} \frac{D_{s}}{\rho n_{0}} \frac{p_{i}-\hat{p}_{j}}{\left|\boldsymbol{r}_{i}-\boldsymbol{r}_{j}\right|^{2}}\left(\boldsymbol{r}_{i}-\boldsymbol{r}_{j}\right) w\left(\left|\boldsymbol{r}_{i}-\boldsymbol{r}_{j}\right|\right)
$$

Since $\left(p_{j}-\hat{p}_{i}\right)\left(\boldsymbol{r}_{j}-\boldsymbol{r}_{i}\right) \neq-\left(p_{i}-\hat{p}_{j}\right)\left(\boldsymbol{r}_{i}-\boldsymbol{r}_{j}\right)$, in general:

$$
\boldsymbol{A}_{j \rightarrow i}^{p} \neq-\boldsymbol{A}_{i \rightarrow j}^{p}
$$

The problem is not caused by the adoption of Eq. 4 proposed by Koshizuka et al. [1998]. Even if $p_{i}$ had not been replaced with the minimum pressure at neighboring particles as in Eq. 4, the pressure interacting forces would have been equal (if $m_{i}=m_{j}$ ) in magnitude but not opposite in direction. Thus, exact conservation of linear momentum will not be guaranteed if the above pressure gradient term is applied. However, linear momentum would be exactly conserved if we derive an anti-symmetric equation for pressure gradient term. By anti-symmetric we mean that pressure gradient calculated between particles $i$ and $j$ (target particle $i$ and its neighboring particle $j$ ) is equal and opposite to that calculated between particles $j$ and $i$ (target particle $j$ and its neighboring particle 
i). In section 4.1, a new anti-symmetric formulation for pressure interacting forces is derived to ensure the exact conservation of linear momentum.

On the contrary to the pressure interacting forces, the preservation of linear momentum is exactly guaranteed for the viscous forces. The viscous force on particle $i$ owing to $j$ is:

$$
\boldsymbol{A}_{j \rightarrow i}^{v}=m_{i} v\left(\nabla^{2} \boldsymbol{u}\right)_{j \rightarrow i}=\frac{2 m_{i} v D_{s}}{\lambda n_{0}}\left(\boldsymbol{u}_{j}-\boldsymbol{u}_{i}\right) w\left(\left|r_{j}-r_{i}\right|\right)
$$

which is exactly equal and opposite to the force on particle $j$ owing to $i$. Therefore, the total sum of all interaction pairs between particles due to viscous accelerations will vanish. Consequently, in case of viscous forces total linear momentum of the system will be preserved.

\subsection{Conservation of angular momentum}

The total angular momentum of a system of particles with respect to the origin is given as:

$$
\boldsymbol{H}=\sum_{i=1}^{N} \boldsymbol{r}_{i} \times m_{i} \boldsymbol{u}_{i}
$$

By time differentiating and considering the law of motion in the absence of external forces, the rate of time change of angular momentum of the system $(=\dot{\boldsymbol{H}})$ will be:

$$
\dot{\boldsymbol{H}}=\sum_{i=1}^{N} \boldsymbol{r}_{i} \times m_{i} \boldsymbol{a}_{i}=-\sum_{i=1}^{N} \boldsymbol{r}_{i} \times \boldsymbol{A}_{i}
$$

Hence, conservation of angular momentum will be guaranteed if the total moment of the internal forces about the origin vanishes, that is:

$$
\sum_{i=1}^{N} \boldsymbol{r}_{i} \times \boldsymbol{A}_{i}=0
$$

Considering again the two neighboring particles shown in Fig. 1, the angular moment of the two interacting forces about the origin can be written as:

$$
\boldsymbol{r}_{i} \times \boldsymbol{A}_{i j}+\boldsymbol{r}_{j} \times \boldsymbol{A}_{j i}
$$

If $\boldsymbol{A}_{i j}=-\boldsymbol{A}_{j i}$, then: 


$$
\boldsymbol{r}_{i} \times \boldsymbol{A}_{i j}+\boldsymbol{r}_{j} \times \boldsymbol{A}_{j i}=-\boldsymbol{r}_{i j} \times \boldsymbol{A}_{i j}
$$

The above term will vanish whenever the interaction force $\boldsymbol{A}_{i j}$ is co-linear with the vector $\boldsymbol{r}_{i j}\left(=\boldsymbol{r}_{j}-\boldsymbol{r}_{i}\right)$. The interacting pressure forces between particles $i$ and $j$ are co-linear with the vector $\boldsymbol{r}_{i j}$ as the pressure term (Eq. 4) is a product of a scalar and the vector $\boldsymbol{r}_{i j}$. However, since the interacting pressure forces are not anti-symmetric (equal in magnitude, opposite in direction), similar to the linear momentum the conservation of angular momentum is not guaranteed. In case of the viscous forces, the interactions do not necessarily lie on the same line with vector $\boldsymbol{r}_{i j}$; thus, the conservation of angular momentum is not guaranteed either. Briefly speaking, in standard MPS method, the angular momentum is not conserved while the linear momentum is conserved only in case of the viscous forces. This fact will be more clearly shown in chapter 5.

\section{Derivation of Corrected MPS (CMPS) Formulations}

\subsection{CMPS: conservation of linear momentum}

As previously discussed in section 3.1, the pressure gradient term in the standard MPS method does not guarantee the conservation of linear momentum. For this reason, we propose another formulation for pressure gradient term. Eq. 4 is rewritten here, splitting the numerator of the fraction containing the pressure terms.

$$
\langle\nabla p\rangle_{i}=\frac{D_{s}}{n_{0}} \sum_{j \neq i}\left\{\frac{p_{j}}{\left|\boldsymbol{r}_{j}-\boldsymbol{r}_{i}\right|^{2}}\left(\boldsymbol{r}_{j}-\boldsymbol{r}_{i}\right) w\left(\left|\boldsymbol{r}_{j}-\boldsymbol{r}_{i}\right|\right)-\frac{\hat{p}_{i}}{\left|\boldsymbol{r}_{j}-\boldsymbol{r}_{i}\right|^{2}}\left(\boldsymbol{r}_{j}-\boldsymbol{r}_{i}\right) w\left(\left(\boldsymbol{r}_{j}-\boldsymbol{r}_{i}\right)\right)\right\}
$$

The concept of the gradient model in the standard MPS and CMPS methods is depicted in Fig. 3. In order to derive the new formulation in an anti-symmetric form, an imaginary point $k$ is considered on the midpoint of the position vector of particle $i$ and its neighboring particle $j$, namely, $\boldsymbol{r}_{i j}$. The gradient term is now modified considering point $k$ and the imaginary position vector $\boldsymbol{r}_{i k}\left(=\boldsymbol{r}_{k}-\boldsymbol{r}_{i}\right)$. 


$$
\langle\nabla p\rangle_{i}=\frac{D_{s}}{n_{0-i k}} \sum_{j \neq i}\left\{\frac{p_{k}}{\left|\boldsymbol{r}_{k}-\boldsymbol{r}_{i}\right|^{2}}\left(\boldsymbol{r}_{k}-\boldsymbol{r}_{i}\right) w\left(\left|\boldsymbol{r}_{k}-\boldsymbol{r}_{i}\right|\right)-\frac{\hat{p}_{i}}{\left|\boldsymbol{r}_{k}-\boldsymbol{r}_{i}\right|^{2}}\left(\boldsymbol{r}_{k}-\boldsymbol{r}_{i}\right) w\left(\left|\boldsymbol{r}_{k}-\boldsymbol{r}_{i}\right|\right)\right\}
$$

In the above equation $n_{0-i k}$ refers to the particle number density in the new imaginary influence circle of particle $i$ which contains the neighboring particles $k$. In the standard MPS method, originally a linear variation of pressure is assumed in the short distance between particle $i$ and its neighboring particle $j$. Hence, $p_{k}$ can be substituted by $\left(p_{j}+p_{i}\right) / 2$ while $\boldsymbol{r}_{i k}$ is also $\boldsymbol{r}_{i j} / 2$. Thus:

$$
\langle\nabla p\rangle_{i}=\frac{D_{s}}{n_{0-i k}} \sum_{j \neq i}\left\{\frac{p_{i}+p_{j}}{\left|\boldsymbol{r}_{j}-\boldsymbol{r}_{i}\right|^{2}}\left(\boldsymbol{r}_{j}-\boldsymbol{r}_{i}\right) w\left(\left|\boldsymbol{r}_{k}-\boldsymbol{r}_{i}\right|\right)-\frac{2 \hat{p}_{i}}{\left|\boldsymbol{r}_{j}-\boldsymbol{r}_{i}\right|^{2}}\left(\boldsymbol{r}_{j}-\boldsymbol{r}_{i}\right) w\left(\left|\boldsymbol{r}_{k}-\boldsymbol{r}_{i}\right|\right)\right\}
$$

On the other hand, it can be shown that the weight function applied in the new imaginary influence circle is equal to the one in the initial influence circle.

$$
w\left(\left|\boldsymbol{r}_{k}-\boldsymbol{r}_{i}\right|\right)=\left(\frac{r_{e-i k}}{r_{i k}}-1\right)=\left(\frac{r_{e-i j} / 2}{r_{i j} / 2}-1\right)=w\left(\left|\boldsymbol{r}_{j}-\boldsymbol{r}_{i}\right|\right)
$$

Therefore, the summation of weight functions in the imaginary influence circle of particle $i$ would be equal to that in the initial influence circle:

$$
n_{0-i k}=\sum_{i \neq k} w\left(\left|r_{k}-r_{i}\right|\right)=\sum_{i \neq j} w\left(\left|r_{j}-r_{i}\right|\right)=n_{0-i j}=n_{0}
$$

Accordingly the new pressure gradient term can be written as:

$$
\langle\nabla p\rangle_{i}=\frac{D_{s}}{n_{0}} \sum_{j \neq i}\left\{\frac{p_{i}+p_{j}}{\left|\boldsymbol{r}_{j}-\boldsymbol{r}_{i}\right|^{2}}\left(\boldsymbol{r}_{j}-\boldsymbol{r}_{i}\right) w\left(\left|\boldsymbol{r}_{j}-\boldsymbol{r}_{i}\right|\right)-\frac{2 \hat{p}_{i}}{\left|\boldsymbol{r}_{j}-\boldsymbol{r}_{i}\right|^{2}}\left(\boldsymbol{r}_{j}-\boldsymbol{r}_{i}\right) w\left(\left|\boldsymbol{r}_{j}-\boldsymbol{r}_{i}\right|\right)\right\}
$$

Since the minimum pressure in the influence circle of particle $i$ is not necessarily equal to that in the influence circle of particle $j$, the above equation is not yet anti-symmetric and needs a slight modification. In order to make it a full anti-symmetric equation, $\hat{p}_{i}$ is replaced by $\left(\hat{p}_{i}+\hat{p}_{j}\right) / 2$. Therefore, the new pressure gradient term in the CMPS method is derived as:

$$
\langle\nabla p\rangle_{i}=\frac{D_{s}}{n_{0}} \sum_{j \neq i} \frac{\left(p_{i}+p_{j}\right)-\left(\hat{p}_{i}+\hat{p}_{j}\right)}{\left|\boldsymbol{r}_{j}-\boldsymbol{r}_{i}\right|^{2}}\left(\boldsymbol{r}_{j}-\boldsymbol{r}_{i}\right) w\left(\left|\boldsymbol{r}_{j}-\boldsymbol{r}_{i}\right|\right)
$$


The linear momentum is exactly conserved when the above anti-symmetric equation is applied. Since the conservation of linear momentum is also guaranteed for the viscous forces (Eq. 20), in the CMPS method the total linear momentum of the system would be exactly conserved.

\subsection{CMPS: conservation of angular momentum}

The exact conservation of angular momentum is not guaranteed in the standard MPS method as the viscous interacting forces are not co-linear with the position vector of two neighboring particles and the pressure interacting forces are not opposite. In the CMPS method the new pressure gradient term is anti-symmetric in addition to being radial (co-linear with the position vector $\boldsymbol{r}_{i j}$ ); hence, angular momentum will be exactly conserved in case of the pressure interacting forces. For the viscous interacting forces, however, conservation of angular momentum is not strictly ensured. A variety of approaches have been introduced in the SPH research by use of which the exact conservation of angular momentum can be guaranteed. Bonet and Lok [1999] proposed a discrete variational approach to ensure the exact preservation of linear and angular momentum. Later, Bonet et al. [2004] introduced the Hamiltonian SPH which also ensures the preservation of linear and angular momentum. Another approach is focused on the completeness of the kernel functions.

The completeness in meshfree methods is equivalent to the consistency in the finite difference literature and refers to the ability of the kernel functions in the exact reproduction of a physical field based on the nodal (particle) values. Liu et al. [1995] proposed a correction function that restores linear (first-order) completeness of the kernel function. In addition to increasing the accuracy [Krongauz and Belytschko, 1997], linear completeness of kernel function also guarantees the global conservation of angular momentum as shown by Belytschko et al. [1998]. However, there are two problems associated with such kind of correction techniques. Firstly, as previously discussed by Bonet and Lok [1999], the use of a first-order (or higher) correction technique would significantly add to the computational time as the coefficients used in correction function are functions of $\boldsymbol{r}_{i j}$. 
Secondly, as the summations are calculated locally over the neighboring particles of a target particle, the correction function computed for particle $i$ is not necessarily equal to that calculated for its neighboring particle $j$. In other words, the anti-symmetric form of the equations is no longer maintained. Vaughan et al. [2008] have also criticized such correction techniques that may violate the anti-symmetric form of equations leading to violation of Newton's third law. Accordingly, linear kernel correction may not always be a promising way of performing more efficient and accurate computations unless an appropriate way for the calculation of such corrective term is chosen. Hence, we postpone the employment of linear kernel correction to near-future works.

\section{Validation of CMPS Method}

In this section, a simple test is carried out to validate the CMPS method and to show its enhanced performance comparing to the standard MPS method. The test is the simulation of an elliptical water drop [Monaghan, 1994; Bonet and Lok, 1999]. The initial fluid configuration is a circle of radius $1 \mathrm{~m}$ subjected to no external forces but an initial velocity field as $(-100 x, 100 y) \mathrm{m} / \mathrm{s}$. During the calculation due to the absence of external forces total linear and angular momentum should be preserved. Moreover, the drop should stay elliptical, the value of $a b$ (semi-minor axis $\times$ semi-major axis) should remain constant (as the fluid is incompressible) and the outer boundary surface should remain smooth. The domain is represented by a total number of 7850 particles being $2 \mathrm{~cm}$ in diameter. The density and viscosity of fluid are chosen equal to those selected in [Bonet and Lok, 1999] that is $\rho=1000 \mathrm{~kg} / \mathrm{m}^{3}$ and $\mu=0.5 \mathrm{~kg} \mathrm{~m}^{-1} \mathrm{~s}^{-1}$. The equation governing the time variation of $b$ is given as [Monaghan, 1994; Bonet and Lok, 1999]:

$$
\frac{\mathrm{d} B}{\mathrm{~d} t}=\frac{B^{2}\left(b^{4}-z^{4}\right)}{\left(b^{4}+z^{4}\right)} ; B=\frac{-1}{b} \frac{\mathrm{d} b}{\mathrm{~d} t}
$$

where $z$ is the initial value of $a b$. The above second order differential equation is solved and the analytical solutions together with the results obtained from both standard MPS and CMPS methods 
are plotted in Fig. 4. Both methods have accurately tracked the evolution of the drop up to $t=0.004$ $s$. From this instant, however, the CMPS method has resulted in a more accurate tracking of the drop outer surface. Fig. 5 shows the time variation of $a b$ which should remain as 1.0 analytically (as the area of the ellipse which is $\pi a b$ should remain equal to that of the initial circle). The numerical error seen from the standard MPS calculation is more than that of the CMPS especially after $t=$ $0.004 \mathrm{~s}$. The snapshots showing the particle configurations at $t=0.008 \mathrm{~s}$ and the initial configuration of particles are depicted in Fig. 6. The CMPS method has resulted in a far smoother outer boundary than the standard MPS. Moreover, the outer boundary near the semi-minor axis $a$ has broken up in the standard MPS snapshot.

Fig. 7 illustrates the time variation of total linear momentum in $x$ and $y$ directions for both standard MPS and CMPS methods. The figure confirms that the conservation of linear momentum is not guaranteed in a standard MPS calculation. On the contrary, total linear momentum of the system is exactly preserved in the CMPS method. The time variation of angular momentum is depicted in Fig. 8. Similar to linear momentum, angular momentum has not remained constant in the standard MPS calculations. On the other hand, the new formulation of pressure gradient term in the CMPS method has significantly improved the conservation of angular momentum. An interesting point which should be noted here is that the emergence of the noticeable numerical errors seen in the standard MPS results (Figs. 4 and 5) is concurrent with the large scale fluctuations of the total linear and angular momentum in the corresponding calculation.

In order to present a clearer image of the momentum conservation in the CMPS method, the time variation of angular momentum and $y$-direction linear momentum are plotted again in Fig. 9 solely for the CMPS calculation. From the figure, the amplitude of fluctuations in total $y$-direction linear momentum remains to be of order of $1 O^{-11}$ all through the calculation. Such small fluctuations are expected to be because of the so-called round-off errors due to machine precision. On the other hand, the amplitude of fluctuations in total angular momentum reaches the order of $1 O^{-4}$ prior to the 
end of calculation. Conclusively, while the linear momentum is exactly conserved, exact conservation of angular momentum is not ensured in a CMPS calculation. Meanwhile, the conservation of angular momentum in the CMPS method is significantly improved as the amplitude of the total angular momentum fluctuatutions seen in the CMPS calculation is about 7500 times less than those in the standard MPS computation.

\section{MPS vs. CMPS Simulation of Solitary Wave Breaking and Post-Breaking on a Slope}

The breaking and post-breaking of nearshore waves are significant phenomena in the field of coastal engineering. Nevertheless, the complexities of fluid motion associated with wave breaking bring about many mathematical difficulties that eliminate the chance of a fully theoretical description. Both of experimental techniques and grid-based numerical simulations suffer from certain constraints and difficulties when they are applied in the study of such violent free-surface flows [see Gotoh et al., 2005 or Shao, 2006 for more details]. On the other hand, particle methods have a significant potential to provide a comprehensive description of the full processes associated with wave breaking, whilst, they can accurately track the water surface all through the wave breaking process. Numerous SPH-based simulations of wave breaking [e.g. Shao, 2006] and post-breaking [e.g. Dalrymple and Rogers, 2006] have been carried out. The MPS method has also been applied in the simulation of wave breaking [e.g. Koshizuka et al., 1998; Gotoh and Sakai, 1999; and Gotoh et al., 2003] and post-breaking [e.g. Khayyer and Gotoh, 2007].

In this section, the CMPS and standard MPS methods are applied in the calculation of solitary wave breaking and post-breaking on a uniform slope. Firstly, the enhanced capability of the CMPS method in the simulation of plunging wave breaking and the resulting post-breaking processes such as the complex process of splash-up is illustrated through qualitative comparisons with laboratory photographs by Li and Raichlen [2003]. After the qualitative comparison, the high accuracy of the CMPS method is further verified by the quantitative comparisons with experimental data by Li 
[2000].

\subsection{Qualitative comparison}

6.1.1 Enhanced reproduction of a plunging breaking wave and resultant splash-up by CMPS method

Shoaling, breaking and post-breaking of a solitary wave with the incident relative wave height or the ratio of offshore wave height $\left(=H_{0}\right)$ to offshore water depth $\left(=h_{0}\right)$ of $H_{0} / h_{0}=0.40$ is simulated over a slope $(=s)$ of 1:15. A schematic view of the computational domain is shown in Fig. 10. The initial offshore water depth is $0.200 \mathrm{~m}$. The particles are $0.005 \mathrm{~m}$ in diameter and about 20000 particles are located in the domain. Since the waves are generated by a moving wall, which initially moves backward and a constant number of particles are employed, by the time when the desired wave is generated the offshore water depth is less than that of the initial one. Hence, numerous preliminary simulations are carried out in order to obtain the desired relative wave height. The incident wave height and the offshore water depth are $0.075 \mathrm{~m}$ and $0.187 \mathrm{~m}$, respectively. The conditions of $H_{0} / h_{0}=0.40$ and $s=1: 15$ lead to a strong plunging breaking in which the plunging jet hits the still water ahead of the wave, consequently a secondary shoreward directed jet is generated from the impact point. Fig. 11 shows three CMPS snapshots of water particles illustrating the initiation of breaking, splash-up initiation and splash-up formation. The particles shown by closed circles are those detected as free-surface particles.

The splash-up is a very complex, yet important process as it is responsible for the generation of large-scale vortices and plays a major role in the dissipation of wave energy and momentum transfer. The complexities of fluid behaviour such as the presence of large deformations and fragmentations have made the simulation of such a process very difficult. The applicability of the standard MPS method in the simulation of splash-up is already shown by Khayyer and Gotoh [2007]. In this section, we show the enhanced capability of the CMPS method in the simulation of a 
strong plunging breaking and resulting splash-up process through the qualitative comparisons with both the standard MPS results and the still photographs taken during the laboratory experiments by Li and Raichlen [2003].

In Fig. 12 the CMPS (left hand side column) and the standard MPS (right hand side column) snapshots are shown together with the laboratory photographs [Li and Raichlen, 2003] located in the middle. By comparing the two series of snapshots with the corresponding laboratory photographs, it is clear that the simulation-experiment qualitative agreement is much better in case of CMPS snapshots. As a result of exact conservation of linear momentum and improved preservation of angular momentum, the CMPS results portray a clearer image of the plunging jet and the air chamber beneath it (Fig. 12(ac-dc)) with less particle scattering as seen in standard MPS results. Moreover, the splash-up is more precisely simulated by the CMPS than the standard MPS. The CMPS method gives a fine reproduction of the splash-up formation (Fig. 12(ec)) matching well with the experiment. The development of the splash-up (Fig. 12(f)) is also well simulated by the CMPS method as the reflected jet angel and the gap between the incident and the reflected jets (Fig. 12(fc)) are in good agreement with the experiment. As the splash-up progresses the reflected jet curls back toward the incident jet and eventually becomes nearly vertical (Fig. 12(g)). This fact is moderately well illustrated in CMPS snapshot (Fig. 12(gc)).

Although the splash-up is much better simulated by the CMPS method, yet, the entire curl of the splash has not been well reproduced (Fig. 12(fc-gc)). One of the main reasons behind this disagreement is expected to be the application of a simplified Laplacian model (Eq. 8) for the treatment of viscosity. In original MPS method, the components of the relative velocity vector between particle $i$ and its neighboring particle $j$ are treated as a scalar quantity and are diffused to neighboring particle $j$ using the Laplacian model. Therefore, it would be difficult for the MPS method to perfectly model a highly non-uniformly deformation of a lump of water such as the splash-up. Later in section 6.1.2, we propose a tensor-type strain-based viscosity term which helps 
the viscous accelerations to be calculated from a strain rate tensor. The Corrected MPS method with a Strain-Based Viscosity will be given the name CMPS-SBV.

Another important issue regarding to the CMPS method is the configuration of free surface and non-free surface particles. Fig. 13 shows the standard MPS and the CMPS snapshots of water particles at the instant of breaking (the instant at which the front face of the wave becomes vertical). In case of the CMPS snapshot, the free surface boundary condition is correctly applied to a thin layer of outer particles representing a smooth free surface. On the contrary, in the standard MPS snapshot the particles detected as free surface do not exclusively lie on the outer layer. This is in direct relation with momentum conservation in the standard MPS method. Since linear momentum is not conserved, the interacting forces between particles are not balanced. Hence, the relative positions of particles might vary significantly in space leading to considerable changes in particle number densities and thus improper detection of free surface particles.

Among the disadvantages associated with the standard MPS method is the problem of artificial pressure fluctuation already addressed by Gotoh et al. [2005]. Because the source term in Poisson pressure equation (Eq. 10) is a direct function of particle number density (and thus relative particle positions), pressure fluctuations seem to be unavoidable in a strictly incompressible particle-based calculation. Meanwhile, the exact conservation of linear momentum in the CMPS method minimizes the fluctuations in the relative positions of inner particles and consequently results in a more realistic instantaneous pressure distribution. Here we show the improved estimation of pressure by the CMPS through a simple test; that is, the time variation of hydrostatic pressure at a fixed point. Fig. 14(a) depicts a schematic view of the computational domain for this test. The MPS and CMPS time histories of pressure at measuring point A are plotted in Fig. 14(b). As previously discussed, the MPS estimation of pressure is accompanied by significant fluctuations with amplitudes being up to four times of the theoretical solution. On the other hand, the CMPS method has resulted in a considerably more realistic instantaneous pressure calculation. 
6.1.2 Further enhanced reproduction of splash-up by CMPS with a Strain-Based Viscosity $(C M P S-S B V)$

In a kernel-based particle method such as the MPS method, the divergence of a function $f(x)$ can be calculated from the following equation:

$$
\left\langle\nabla \cdot f\left(x_{i}\right)\right\rangle=\sum_{j \neq i}^{N_{i}} V_{j} f\left(x_{j}\right) \cdot \nabla_{i} w_{i j}
$$

Therefore:

$$
\left(v \nabla^{2} \boldsymbol{u}\right)_{i}=\left(\frac{1}{\rho} \nabla \cdot \boldsymbol{T}\right)_{i}=\frac{1}{\rho} \sum_{j \neq i}^{N_{i}} V_{j} \boldsymbol{T}_{i j} \cdot \nabla_{i} w_{i j}=\frac{1}{\rho n_{0}} \sum_{j \neq i}^{N_{i}} \boldsymbol{T}_{i j} \cdot \nabla_{i} w_{i j}
$$

where $\boldsymbol{T}=$ the viscous stress tensor which can be related to the strain rate of flow by the following equation:

$$
\boldsymbol{T}_{i j}=2 \mu \boldsymbol{S}_{i j} \quad ; \quad \boldsymbol{S}_{i j}=\left[\begin{array}{lc}
\left(\frac{\partial u}{\partial x}\right)_{i j} & \left(\frac{1}{2}\left[\frac{\partial u}{\partial y}+\frac{\partial v}{\partial x}\right]\right)_{i j} \\
\left(\frac{1}{2}\left[\frac{\partial u}{\partial y}+\frac{\partial v}{\partial x}\right]\right)_{i j} & \left(\frac{\partial v}{\partial y}\right)_{i j}
\end{array}\right]
$$

In Eq. 36, $\mu=$ dynamic viscosity; $u$ and $v=$ the components of the particle velocity in $x$ and $y$ directions, respectively. The velocity and kernel gradients are introduced for each particle as:

$$
\begin{gathered}
\left(\frac{\partial u}{\partial x}\right)_{i j}=\left(\frac{\partial u}{\partial r} \frac{\partial r}{\partial x}\right)_{i j}=\frac{u_{i j}}{r_{i j}} \frac{x_{i j}}{r_{i j}}=\frac{u_{j}-u_{i}}{r_{i j}} \frac{x_{j}-x_{i}}{r_{i j}} \\
\nabla_{i} w_{i j}=\left(\frac{\partial w_{i j}}{\partial x}\right)_{i} \boldsymbol{i}+\left(\frac{\partial w_{i j}}{\partial y}\right)_{i} \boldsymbol{j}=\left(\frac{\partial w_{i j}}{\partial r} \frac{\partial r}{\partial x}\right)_{i} \boldsymbol{i}+\left(\frac{\partial w_{i j}}{\partial r} \frac{\partial r}{\partial y}\right)_{i} \boldsymbol{j}=\frac{-r_{e} x_{i j}}{r_{i j}^{3}} \boldsymbol{i}+\frac{-r_{e} y_{i j}}{r_{i j}^{3}} \boldsymbol{j}
\end{gathered}
$$

The strain-based viscosity introduced above (Eq. 35) exactly preserves linear momentum; yet, similar to the original MPS formulation of viscosity (Eq. 8), it does not exactly conserve angular momentum. Fig. 15 shows the snapshots of standard MPS, CMPS, and CMPS with Strain-Based Viscosity (CMPS-SBV) and the laboratory photographs [Li and Raichlen, 2003]. The employment 
of a strain-based viscosity has resulted in an enhanced reproduction of the entire stages of splash-up formation (Fig. 15(e)), development (Fig. 15(f)) and its curling back (Fig. 15(g)). From Fig. 15(e), the geometrical shape of the plunging jet and the air chamber beneath it are in better agreement with the experiment. In both CMPS and standard MPS snapshots the thickness of the plunging jet at the time of splash-up formation seems to be more uniform than what is seen in the experiment. A precise reproduction of the splash-up development is illustrated by the CMPS-SBV (Fig. 15(f)) without the excessive dispersiveness of particles seen in CMPS and standard MPS snapshots. The curling back of the splash-up is finely simulated by the CMPS-SBV (Fig. 15(g)) as the reflected jet angel, the gap between the plunging jet and the reflected jet and the geometrical shape of the air chamber are in very good agreement with the experiment.

\subsection{Quantitative comparison}

In order to further evaluate the accuracy of the proposed CMPS method, another case of solitary plunging breaking is simulated and the CMPS and standard MPS simulation results are quantitatively compared to the corresponding experimental data by $\mathrm{Li}$ [2000]. Quantitative comparisons are made in terms of wave breaking characteristics such as variation in wave-height, geometrical properties of plunging jet and horizontal velocity of the tip of the plunging jet. The physical conditions of the simulations are equivalent to those in section 6.1 except for the incident wave characteristics. For both the CMPS and standard MPS simulations the incident wave height and the offshore water depth are $0.057 \mathrm{~m}$ and $0.189 \mathrm{~m}$, respectively. Hence, shoaling, breaking and post-breaking of a solitary wave with relative wave height of $H_{0} / h_{0}=0.30$ is simulated over a slope of 1:15. Some CMPS (left) and standard MPS (right) snapshots together with the horizontal velocity field are shown in Fig. 16. In this figure $t_{\mathrm{b}-\mathrm{MPS}}$ and $t_{\mathrm{i}-\mathrm{MPS}}$ correspond to the breaking and plunging jet impact instants in standard MPS calculation, while the breaking and impact instants in CMPS calculation are denoted by $t_{\mathrm{b}-\mathrm{CMPS}}$ and $t_{\mathrm{i} \text {-CMPS, }}$, respectively. It should be noted that in Fig. 16 the time 
interval of the plots for the CMPS and that for the standard MPS are not the same.

Fig. 17 shows a comparison of the variation in wave height $H / H_{0}$ among the CMPS and standard MPS results and experimental data $[\mathrm{Li}, 2000]$. In the figure the ratio $h_{0} / h_{1}$ represents the ratio of the offshore water-depth $\left(=h_{0}\right)$ to the local depth $\left(=h_{1}\right)$. From Fig. 17 it is evident that the CMPS method has resulted in a more accurate prediction of the change in wave height during breaking. Compared to the CMPS, the standard MPS results have shown large fluctuations in predicting the wave height variation curve. One point that should be noted here is that fully isolated particles are not taken into account in the estimation of wave height as well as other quantitative indicators mentioned afterwards. A fully isolated particle is considered as a particle for which there is no neighboring particle (like the one seen in standard MPS snapshot near the wave front in Fig. 13). Meanwhile, for a semi-isolated particle, i.e. an isolated particle (from the rest of particles) for which there is at least one neighboring particle (like the one seen in Fig. 16 in $t_{\mathrm{b}-\mathrm{CMPS}}$ snapshot) the quantitative indicators are measured using the midpoint of a semi-isolated particle and its neighboring particle(s).

The variation of the normalized horizontal velocity of the plunging jet tip is plotted in Fig. 18 . In this figure, $x_{t}$ and $x_{b}$ indicate the $x$-coordinate of plunging jet tip and the breaking point, respectively. The experimental data are obtained from the high-speed video images by dividing the $x$ coordinate of the tip in consecutive images by the time interval between frames [Li, 2000]. A relatively large variation can be seen in the plotted experimental data, which is expected to be caused by the accuracy limitation of the high-speed video [Li, 2000]. However, the experimental data indicate that the horizontal velocity of the plunging jet tip is nearly constant over most of the jet trajectory. Both CMPS and standard MPS results agree with the experiment, while, they do not contain large variations like those seen in the experimental data.

Another quantitative comparison which can further highlight the accuracy of the CMPS method in the tracking of water surface during plunging wave breaking is the geometrical properties of the 
plunging jet. Li [2000] utilized the following characteristics to describe the plunging jet: (i) trajectory of the tip of the plunging jet, and (ii) the length and thickness of the jet prior to impingement. The CMPS and standard MPS predictions of the trajectory of the plunging jet tip are depicted in Fig. 19 together with the experimental data [Li, 2000]. Compared to the standard MPS, the CMPS has given a much more accurate prediction of the motion and location of the plunging jet tip.

The length and thickness of the plunging jet are defined by means of three geometrical properties equivalent to the ones specified by $\mathrm{Li}$ [2000]. The length of the jet $L_{1}$ is defined as the horizontal distance from the tip of the jet to the nearest location of the wave surface which is vertical, as shown in Fig. 20. Two other parameters are used to describe the thickness of the jet; one is the thickness of the jet at the wave vertical plane $\left(=L_{2}\right)$, and the other one $\left(=L_{3}\right)$ is the thickness of the jet at half length of the jet or $L_{1} / 2$. These three parameters do not only describe the geometrical properties of the jet, but also portray an image of the time and spatial evolution of the wave shape during the development of the plunging jet.

From Fig. 21(a), it can be seen that the variation of the plunging jet length $L_{1}$ is considerably better anticipated by the CMPS than the standard MPS. In addition, the CMPS-predicted length of the jet at the impingement instant is nearly equal to the experimental data. The variations of $L_{2}$ and $L_{3}$ are depicted in Fig. 21(b-c). From Fig. 21(b), both models have overestimated the length $L_{2}$, yet, the overestimations seen in the standard MPS results is more than those observed in the CMPS data. The CMPS prediction of the jet thickness $L_{3}$ is in better agreement with the experimental data (Fig. 21(c)). Fig. 21(b-c) also indicates that the lengths $L_{2}$ and $L_{3}$ remain nearly constant during the development of the plunging jet and the thickness of the jet at the middle $\left(=L_{3}\right)$ is about half of that at the base of the jet $\left(=L_{2}\right)$.

Comparing Figs. 19 and 21(a) with Fig. 18, it can be seen that while the trajectory and length of the jet $\left(=L_{1}\right)$ is better predicted by the CMPS than by the standard MPS, the horizontal velocity of 
the jet tip is nearly the same in both methods. Such a difference in prediction can be clarified from Figs. 16 and 17. From these two figures, the wave breaking in standard MPS has happened in a deeper water depth compared to CMPS. While the breaking point in standard MPS $\left(x_{\mathrm{b}-\mathrm{MPS}}=3.74 \mathrm{~m}\right)$ is less than that in CMPS $\left(x_{\mathrm{b}-\mathrm{CMPS}}=3.86 \mathrm{~m}\right)$, the jet impact point is nearly the same in both methods. Accordingly, the plunging jet in standard MPS has traveled a longer distance, in a greater time, with a horizontal velocity being about equal to that in CMPS.

\section{CPU Time for MPS and CMPS Calculations of Wave Breaking and Post-Breaking}

The numerical computations of the current paper are carried out by a single CPU Intel Core ${ }^{\mathrm{TM}} 2$ Duo $2.40 \mathrm{GHz}$ with $2.00 \mathrm{~GB}$ system memory. In all the performed wave breaking and post-breaking computations in chapter 6 , the calculation time step is set according to Courant stability condition and a time resolution chosen as $5.0 \times 10^{-4}$ seconds, that is:

$$
\Delta t=\min \left(\alpha_{\mathrm{dt}} d_{0} / u_{\max }, 5.0 \times 10^{-4}\right)
$$

where $\alpha_{\mathrm{dt}}=$ ratio of the time step to Courant number $(=0.1) ; u_{\max }=$ instantaneous maximum velocity of particles; and $d_{0}=$ particle diameter. The calculations are ended at simulation time of $t=8.800$ seconds. The total CPU times required for the MPS, CMPS, and CMPS-SBV calculations are $12.638,12.349$ and 12.356 hours, respectively. The CPU time of principal routines per time step for the MPS, CMPS, and CMPS-SBV methods is shown in Fig. 22. In both CMPS and CMPS-SBV methods, the introduction of a new formulation for pressure term has slightly increased the CPU time for some pressure calculation processes including the pressure gradient calculation. The number of iterations (and accordingly the required CPU time) for pressure convergence is nearly the same in all the methods. However, as a result of momentum conservation, the computational time required for adjustment of particle positions to maintain the stability of the code is reduced in case of CMPS and CMPS-SBV. This reduction in computational effort leads to slightly less CPU time in 
case of the CMPS and CMPS-SBV. The CPU time for CMPS-SBV is slightly more than that of the CMPS mainly due to the employment of a tensor-type viscosity. Consequently, the CMPS and CMPS-SBV have resulted in more accurate computation of wave breaking and post-breaking, while, the computational time for these methods is nearly the same as in the standard MPS.

\section{Conclusive Remarks}

The paper presents a Corrected Moving Particle Semi-implicit (CMPS) method for the accurate tracking of water-surface during wave breaking and post-breaking. The momentum conservation properties of standard MPS formulations are discussed. A new formulation for pressure gradient term is derived and proposed for the exact (and nearly exact) conservation of linear (and angular) momentum in a MPS-based calculation. The highly improved performance of the new method is shown by a simple test, that is, the evolution of an elliptical water drop. The CMPS method is applied to the simulation of wave breaking and post-breaking on a plane slope. The significantly improved accuracy of the CMPS method in the simulation of such highly non-uniformly deformed flow is confirmed through both qualitative and quantitative comparisons with experimental data and standard MPS results. A tensor-type strain-based viscosity is proposed when the CMPS method is applied to simulate a highly non-uniformly deformed flow such as the splash-up. Further enhanced reproduction of splash-up obtained from the CMPS-SBV (CMPS with Strain-Based Viscosity) method is presented with the results being qualitatively compared to the experiment.

The present study highlights the importance of momentum conservation in a particle-based calculation of free-surface flows. However, in addition to the momentum conservation, several other issues should be considered in a particle-based simulation especially when highly accurate results are desired. Many of such key issues have already been addressed and discussed by Gotoh and Sakai [2006] in details. The step-by-step extension of CMPS method to a 3D multi-phase code with the Sub-Particle-Scale (SPS) turbulence modeling [Gotoh et al., 2001b] is among the future 
works.

\section{References:}

Belytschko, T., Krongauz, Y., Dolbow, J. and Gerlach, C. [1998] "On the completeness of meshfree particle methods," Int. J. Numer. Meth. Eng. 43, 785-819.

Bonet, J. and Lok, T. S. [1999] "Variational and momentum preservation aspects of smooth particle hydrodynamic formulation," Comput. Meth. Appl. Mech. Eng. 180, 97-115.

Bonet, J., Kulasegaram, S., Rodriguez-Paz, M.X., Profit, M. [2004] "Variational formulation for the smooth particle hydrodynamics (SPH) simulation of fluid and solid problems," Comput. Meth. Appl. Mech. Eng. 193(12), 1245-1256.

Dalrymple, R.A. and Rogers, B.D. [2006] "Numerical modeling of water waves with the SPH method," Coastal Eng. 53, 141-147.

Gotoh, H., Hayashi, M., Sakai, T. and Oda, K. [2003] "Numerical Model of Wave Breaking by Lagrangian Particle Method with Sub-Particle-Scale Turbulence Model," Proc. APAC. 2003, Makuhari, Japan.

Gotoh, H., Ikari, H., Memita, T. and Sakai, T. [2005] "Lagrangian particle method for simulation of wave overtopping on a vertical seawall," Coast. Eng. J. 47(2 \& 3), 157-181.

Gotoh, H. and Sakai, T. [1999] "Lagrangian simulation of breaking waves using particle method," Coast. Eng. J. 41 (3 \& 4), 303-326.

Gotoh, H. and Sakai, T. [2006] "Key Issues in the Particle Method for Computation of Wave Breaking," Coastal Eng. 53(2-3), 171-179.

Gotoh, H., Sakai, T. and Hayashi, M. [2001a] "Lagrangian Two-Phase Flow Model for the Wave Generation Process due to Large-Scale Landslides," Proc. APCE 2001, Dalian, China, 176-185.

Gotoh, H., Shibahara, T. and Sakai, T. [2001b] "Sub-Particle-Scale Turbulence Model for the MPS Method -Lagrangian Flow Model for Hydraulic Engineering," Comput. Fluid Dyn. J. 9(4), 339-347.

Harlow, F. H. and Welch, J. E. [1965] "Numerical calculation of time-dependent viscous incompressible 
flow of fluid with free surface," Phys. Fluid, 8, 2182-2189.

Hirt, C. and Nichols, B. D. [1981] "Volume of fluid (VOF) method for the dynamics of free boundaries," J. Comput. Phys. 39, 201-225.

Khayyer, A. and Gotoh, H. [2007] "Applicability of MPS method to breaking and post-breaking of solitary waves," Annual Journal of Hydraulic Engineering, JSCE 51, 175-180.

Koshizuka, S., Tamako, H. and Oka, Y. [1995] “A particle method for incompressible viscous flow with fluid fragmentation," Comput. Fluid Dyn. J. 4(1), 29-46.

Koshizuka, S. and Oka, Y. [1996] "Moving particle semi-implicit method for fragmentation of incompressible fluid," Nuclear Science and Engineering, 123, 421-434.

Koshizuka, S., Nobe, A. and Oka, Y., [1998] "Numerical Analysis of Breaking Waves Using the Moving Particle Semi-implicit Method," Int. J. Numer. Meth. Fluid, 26, 751-769.

Koshizuka, S., Chikazawa, Y. and Oka Y. [2001a] "A particle method for elastic and visco-plastic structures and fluid-structure interactions," Comput. Mech. 27, 97-106.

Koshizuka, S. and Oka, Y. [2001b] "Application of Moving Particle Semi-implicit Method to Nuclear Reactor Safety," Comput. Fluid Dyn. J. 9, 366-375.

Krongauz, Y. and Belytschko, T. [1997] "Consistent pseudo derivatives in meshless methods," Comput. Meth. Appl. Mech. Eng. 146, 371-386.

Li, Y. [2000] "Tsunamis: Non-breaking and breaking solitary wave run-up," Rep. KH-R-60, W. M. Keck Laboratory of Hydraulics and Water Resources, California Institute of Technology, Pasadena, CA.

Li, Y. and Raichlen, F. [2003] "Energy balance model for breaking solitary wave runup," J. Wtrwy. Port Coast. Oc. Eng., ASCE 129(2), 47-59.

Liu, W., Adee, J. and Jun, S. [1993] "Reproducing kernel and wavelets particle methods for elastic and plastic problems," Advanced Computational Methods for Material Modeling, AMD 180/PVP 268 ASME, 175-190.

Liu, W., Jun, S., Li, S., Adee, J. and Belytschko, T. [1995] "Reproducing kernel particle methods for 
structural dynamics," Int. J. Numer. Meth. Eng. 38, 1655-1680.

Monaghan, J. J. [1994] “Simulating free surface flows with SPH,” J. Comput. Phys. 110, 399-406.

Shao, S.D. [2006] "Simulation of breaking wave by SPH method coupled with $k-\varepsilon$ model," Journal of Hydraulic Research, IAHR 44(3), 338-349.

Suzuki, Y., Koshizuka, S. and Oka, Y. [2007] "Hamiltonian moving-particle semi-implicit (HMPS) method for incompressible fluid flows," Comput. Meth. Appl. Mech. Eng. 196(29-30), 2876-2894.

Tsubota, K., Wada, S., Kamada, H., Kitagawa, Y., Lima, R., and Yamaguchi, T. [2006] "A Particle Method for Blood Flow Simulation, -Application to Flowing Red Blood Cells and Platelets-," Journal of the Earth Simulator, 5, 2-7.

Vaughan, G. L., Healy, T. R., Bryan, K. R., Sneyd, A. D. and Gorman, R. M. [2008] "Completeness, conservation and error in SPH for fluids," Int. J. Numer. Meth. Fluids, 56, 37-62.

Yabe, T., Ishikawa, T., Wang, P. Y., Aoki, T., Kadota, Y. and Ikeda, F. [1991] “A Universal Solver for Hyperbolic Equations by Cubic-Polynomial Interpolation - II. Two- and Three- Dimensional Solvers,” Computer Physics Communications, 66, 233-242.

Yabe, T., Xiao, F., Utsumi, T. [2001] "The constrained interpolation profile method for multiphase analysis," J. Comput. Phys. 169, 556-593. 


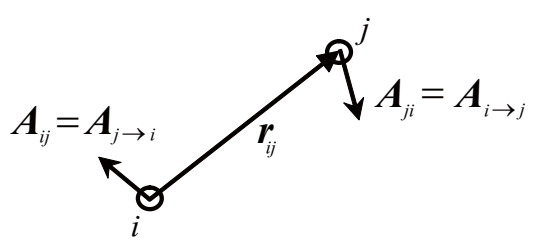

Fig. 1. Internal interaction forces between two neighboring particles

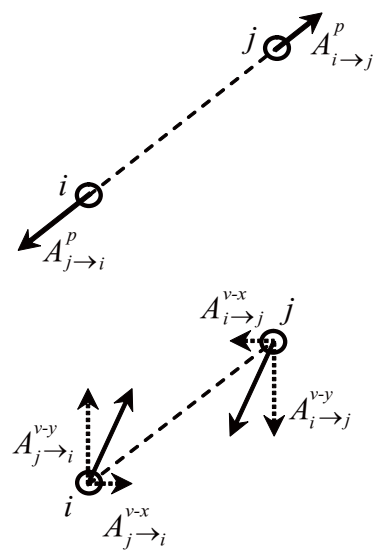

(a)

(b)

Fig. 2. Decomposition of internal interaction forces between two neighboring particles - (a) internal forces due to pressure (b) internal forces due to viscosity

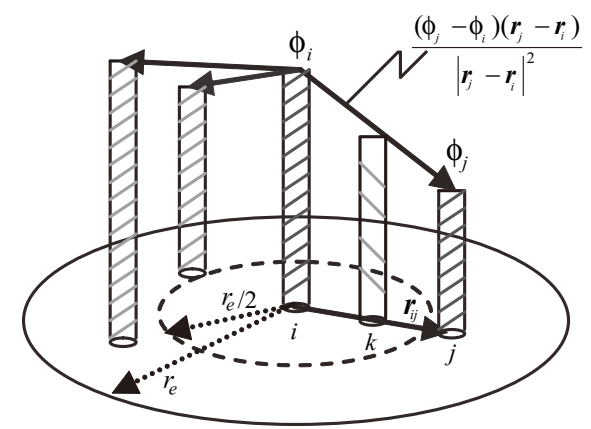

Fig. 3. Concept of gradient operator in standard MPS and CMPS methods 


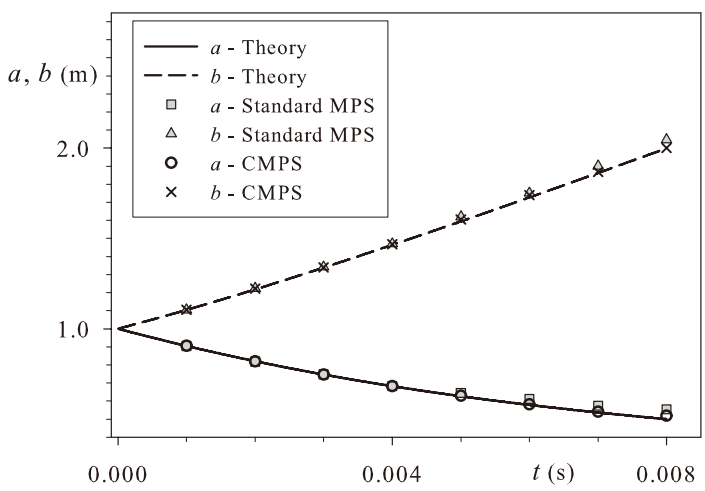

Fig. 4. Computational and theoretical variation of the elliptical drop semi-minor $(a)$ and semi-major $(b)$ axis's

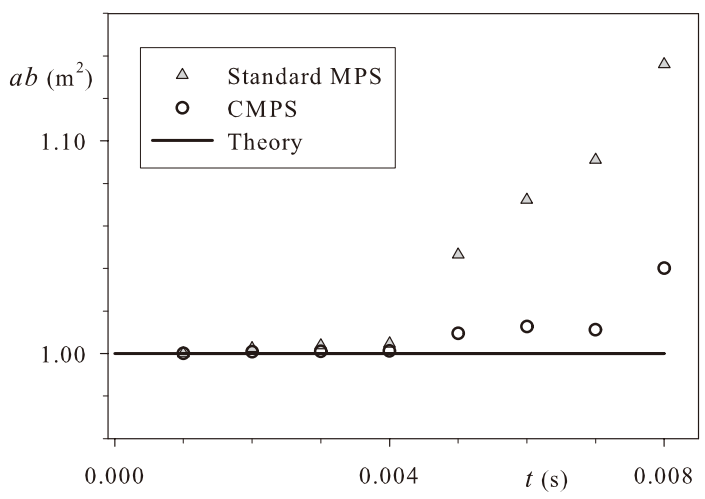

Fig. 5. Computational and theoretical variation of the elliptical drop axis production $(a b)$
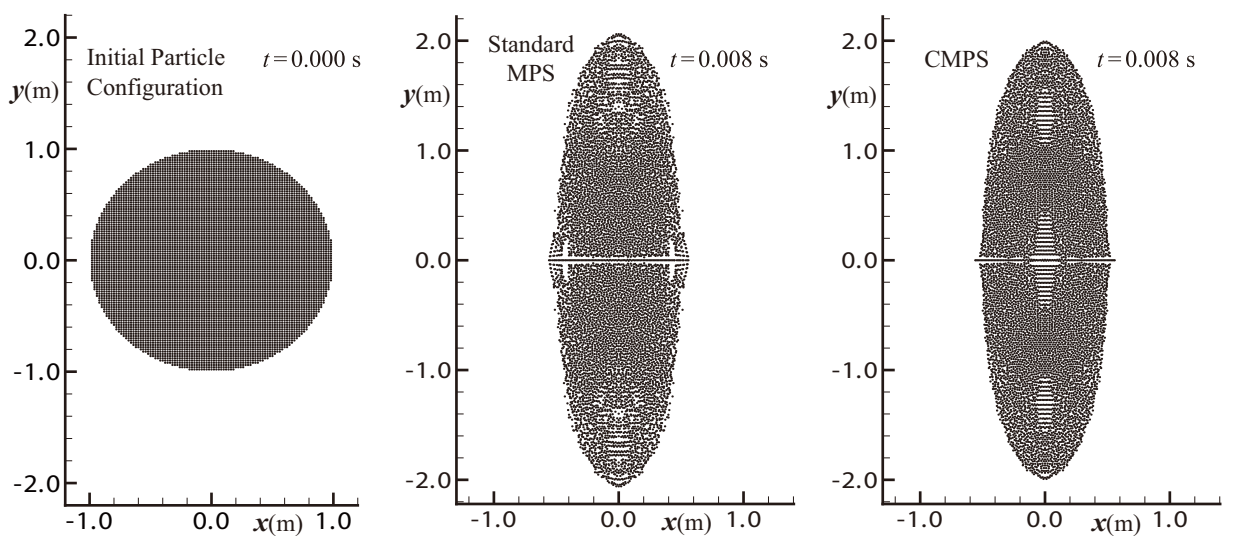

Fig. 6. Standard MPS (middle) and CMPS (right) particle configurations for the evolution of an elliptical drop as it evolves from a circle (left) to a narrow ellipse 

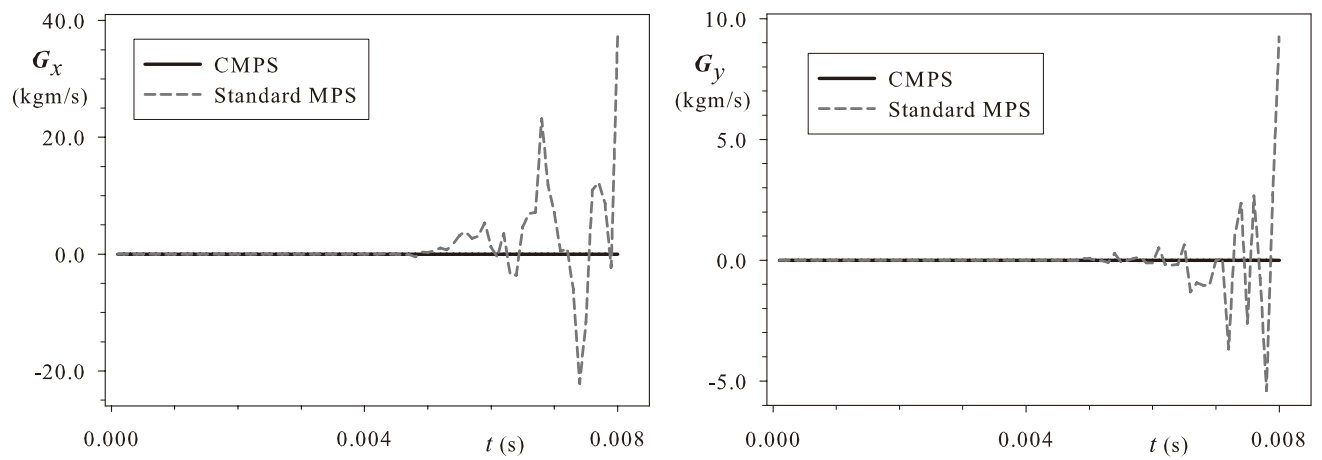

Fig. 7. Variation of total $x$-direction (left) and $y$-direction (right) linear momentum during the evolution of the elliptical drop

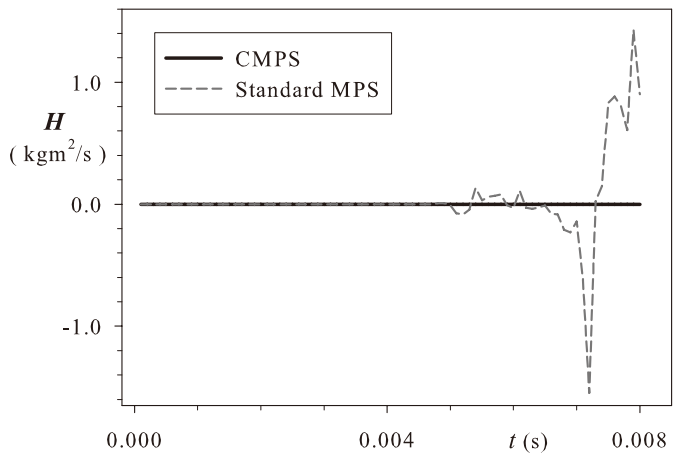

Fig. 8. Variation of total angular momentum during the evolution of the elliptical drop
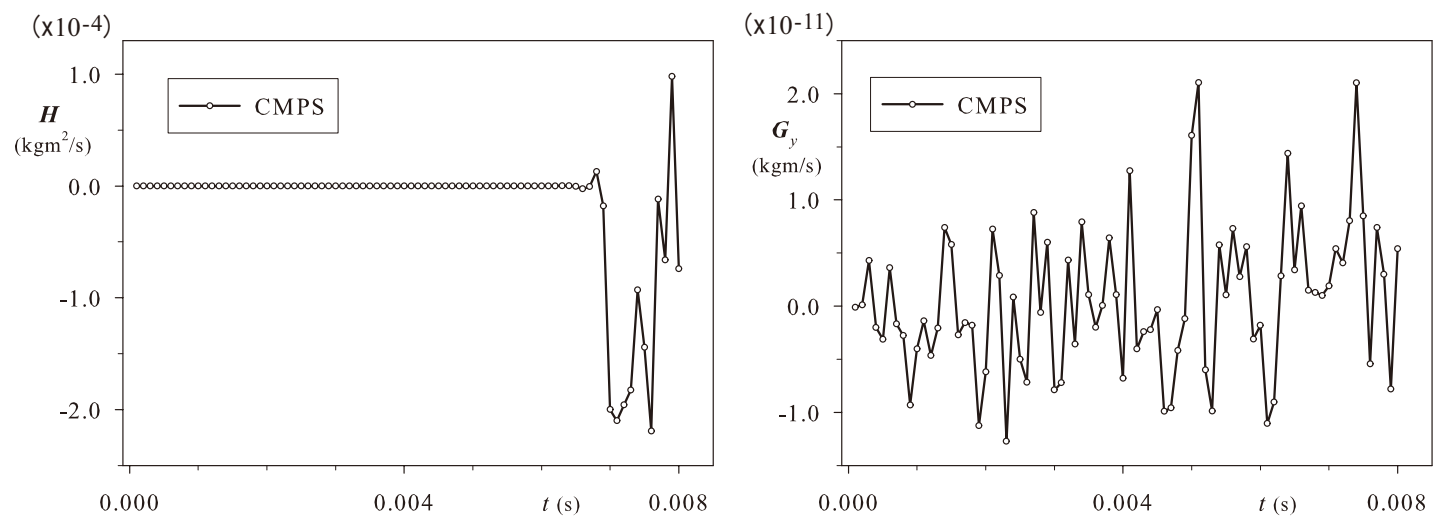

Fig. 9. CMPS variation of total angular momentum (left) and $y$-direction linear momentum (right) during the evolution of the elliptical drop 


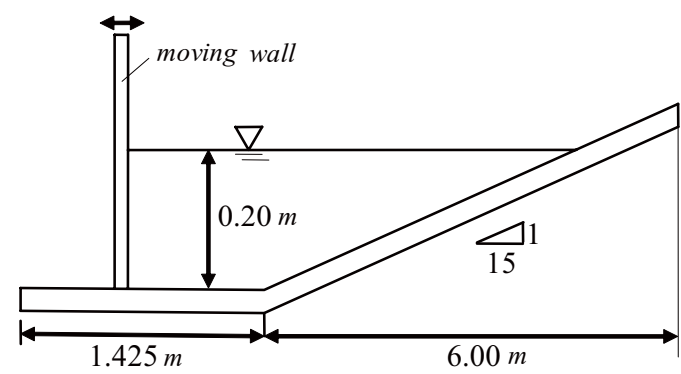

Fig. 10. Sketch of the computational domain for wave breaking and post-breaking simulations
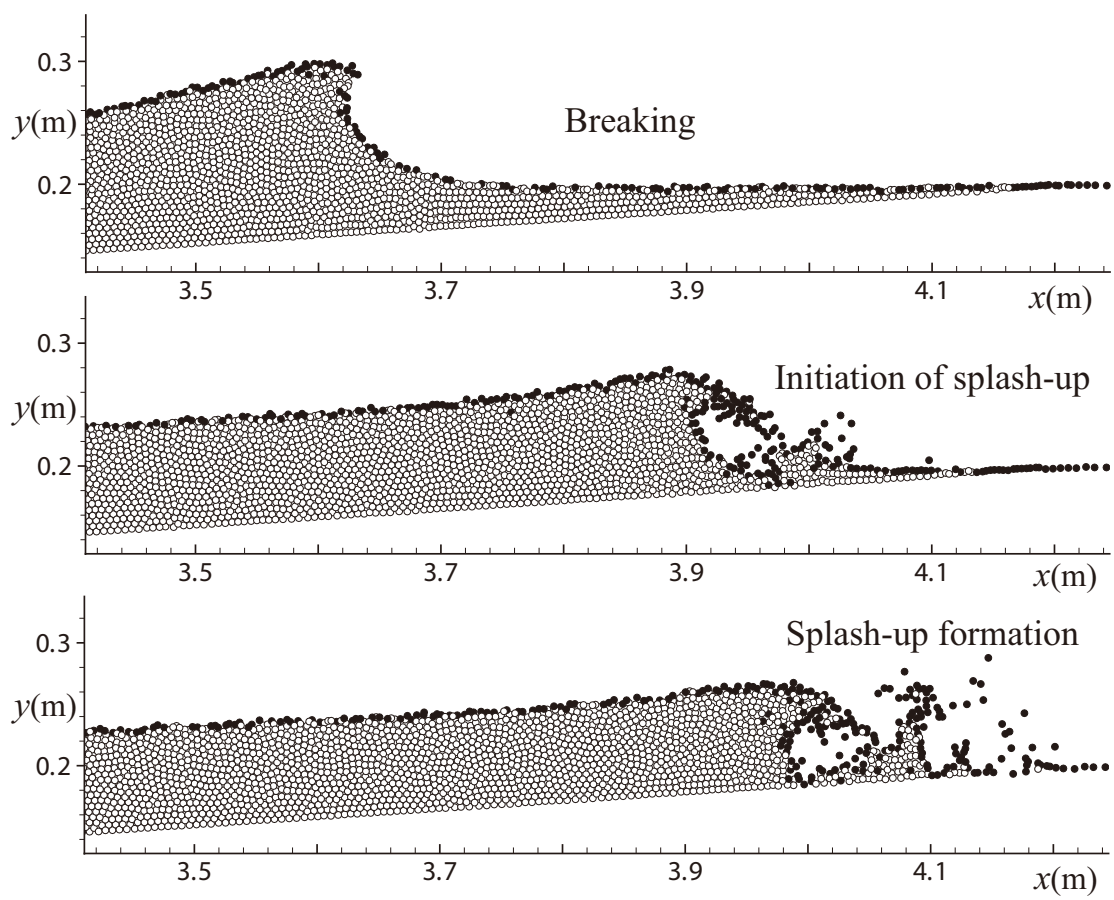

Fig. 11. CMPS snapshots illustrating a strong plunging wave breaking and resulting splash-up $\left(H_{0} / h_{0}=0.40\right)$ 
(ac)
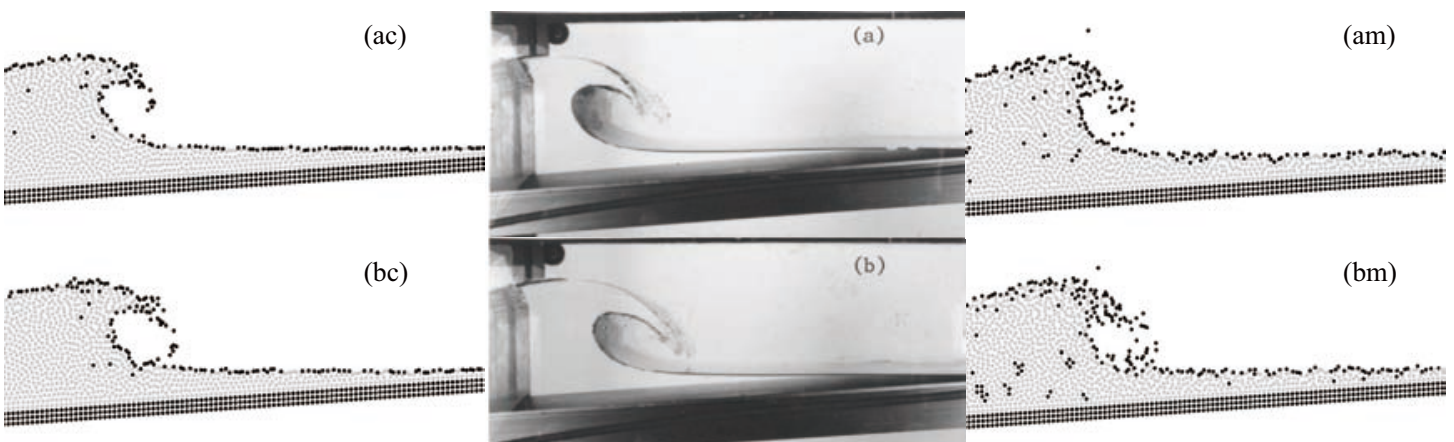

H
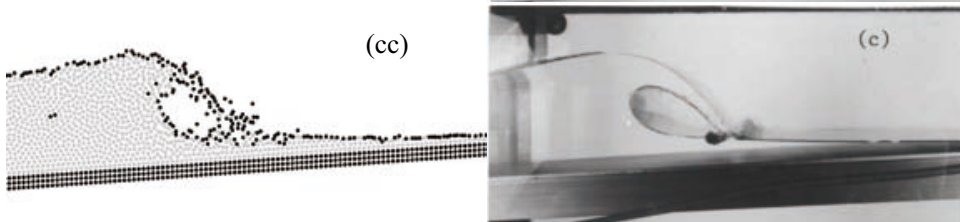

c)<smiles>[As]=C=[W]=[W]</smiles>

(bm)

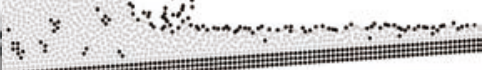
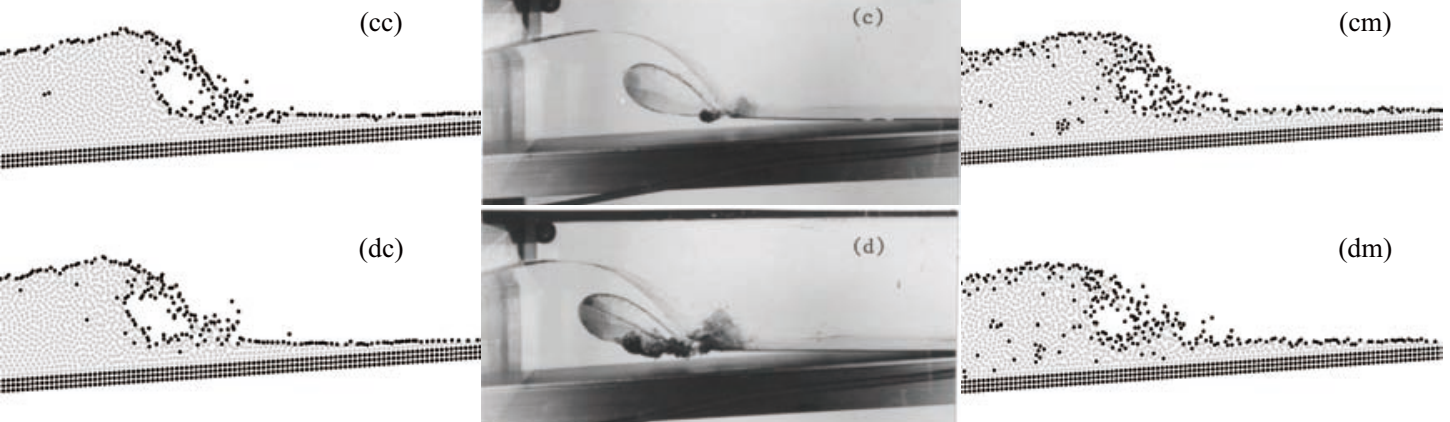

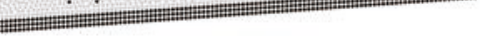
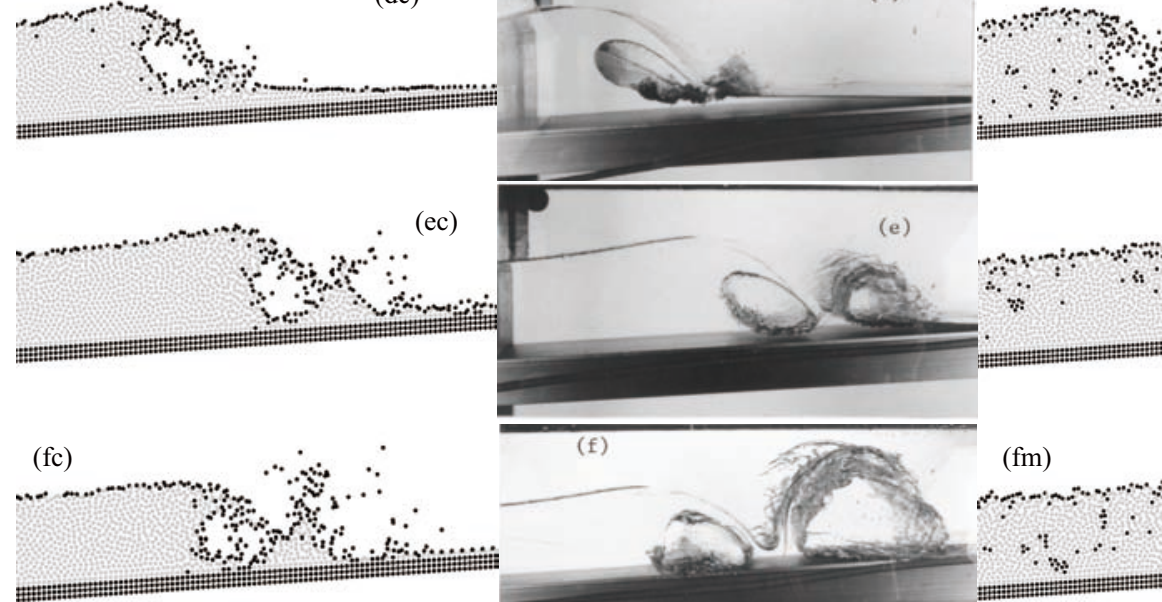

$(\mathrm{dm})$

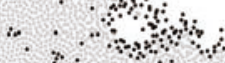

. $3.8+\%$, L.

(gc)

(g)

(f)
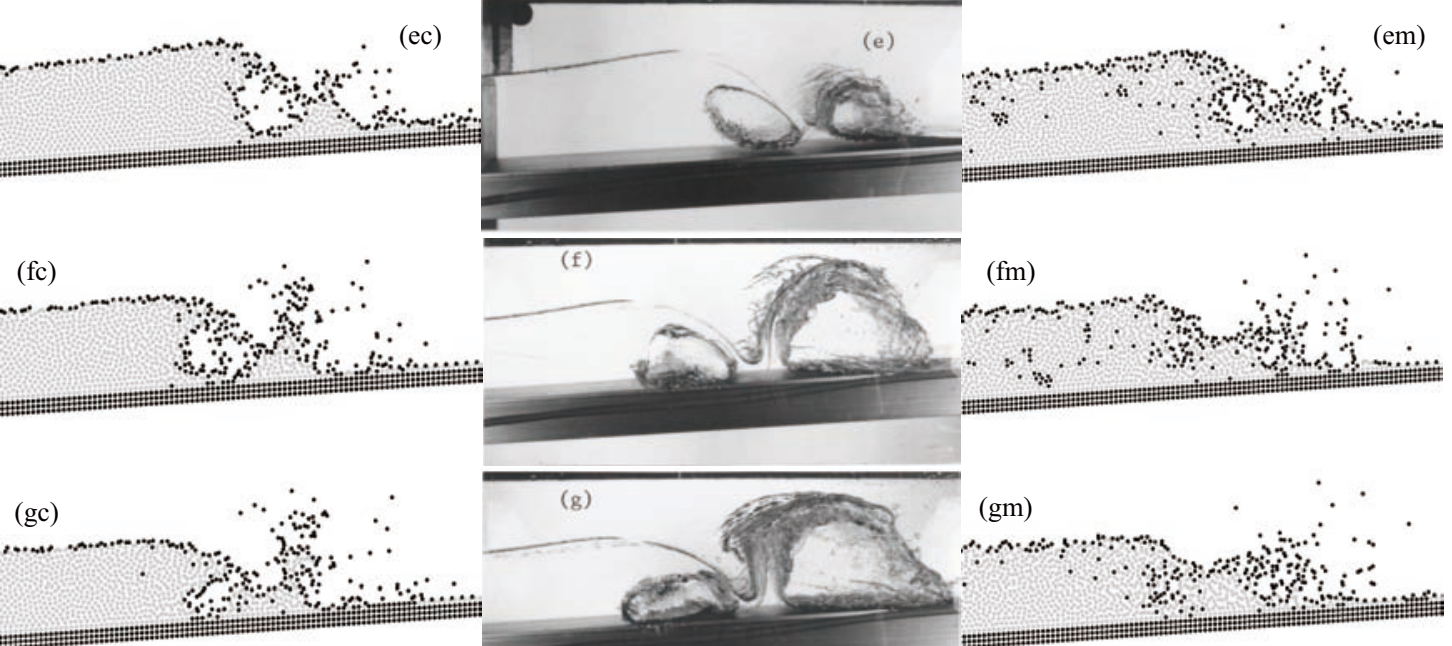

(fm)

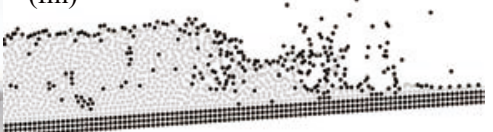
4

(gm)

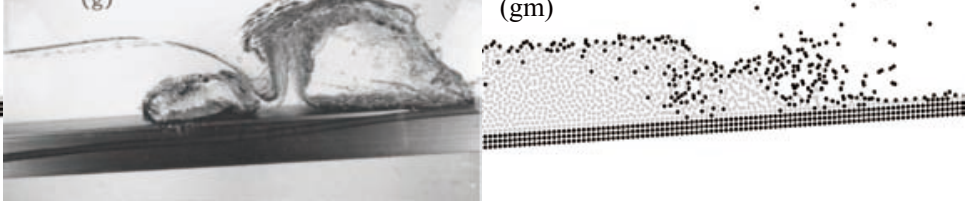

Fig. 12. Strong plunging wave breaking and resulting splash-up $\left(H_{0} / h_{0}=0.40\right)$ - qualitative comparison of laboratory photographs (center; Li and Raichlen [2003]) with CMPS (left) and standard MPS (right) snapshots 
- Particles detected as free-surface

- Non-free surface particles
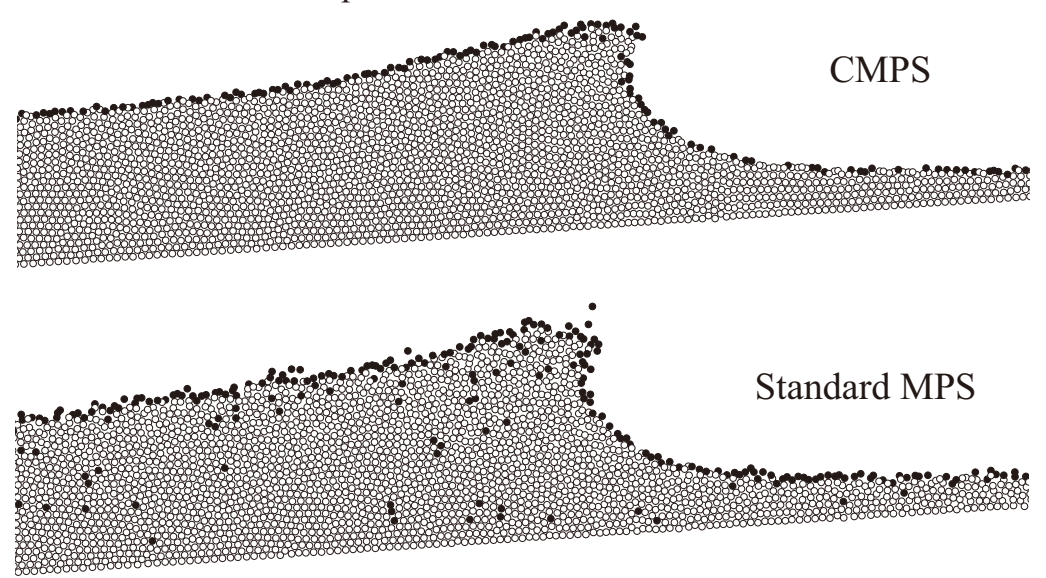

Fig. 13. Configuration of free surface and non-free surface particles in CMPS and standard MPS calculations
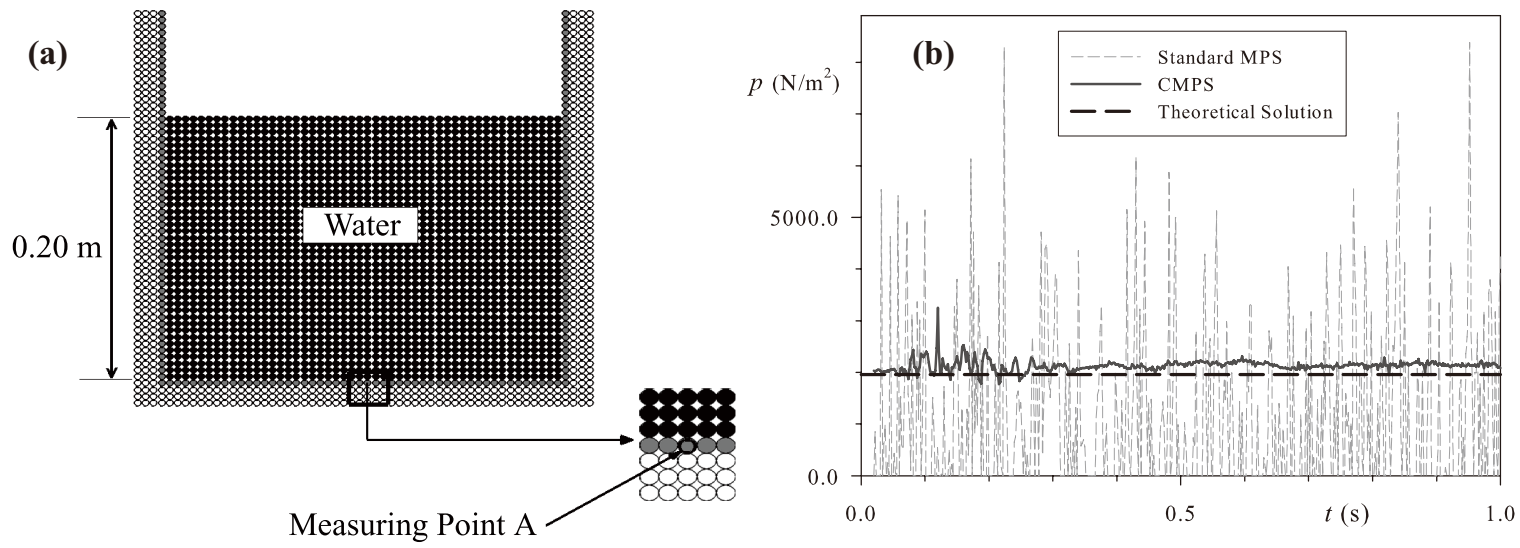

Fig. 14. Improved calculation of instantaneous pressure by CMPS (a) schematic description of the test (b) time histories of hydrostatic pressure at measuring point $\mathrm{A}$ 

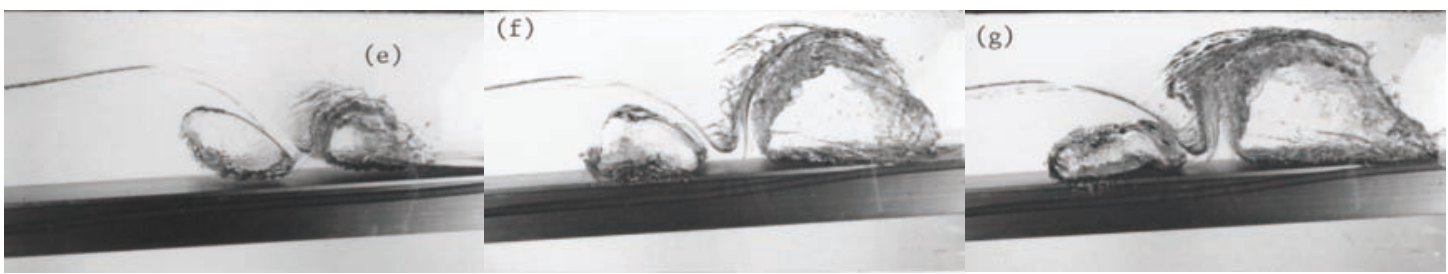

(e) CMPS-SBV
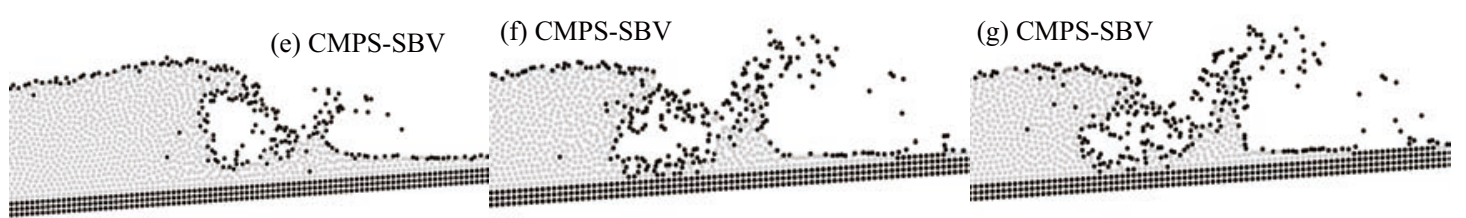

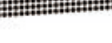

\section{(e) CMPS}

(f) CMPS

(g) CMPS

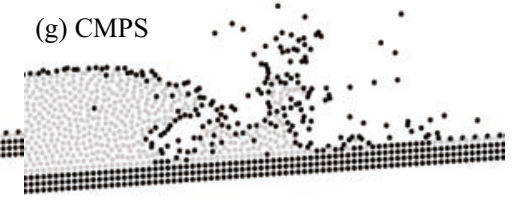
(n)

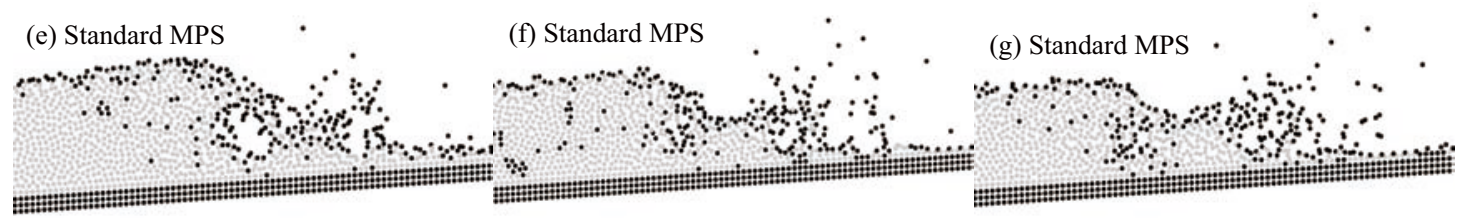

Fig. 15. Further enhanced reproduction of splash-up - qualitative comparison of laboratory photographs (Li and Raichlen [2003]) with CMPS-SBV (CMPS with Strain-Based Viscosity), CMPS and standard MPS snapshots
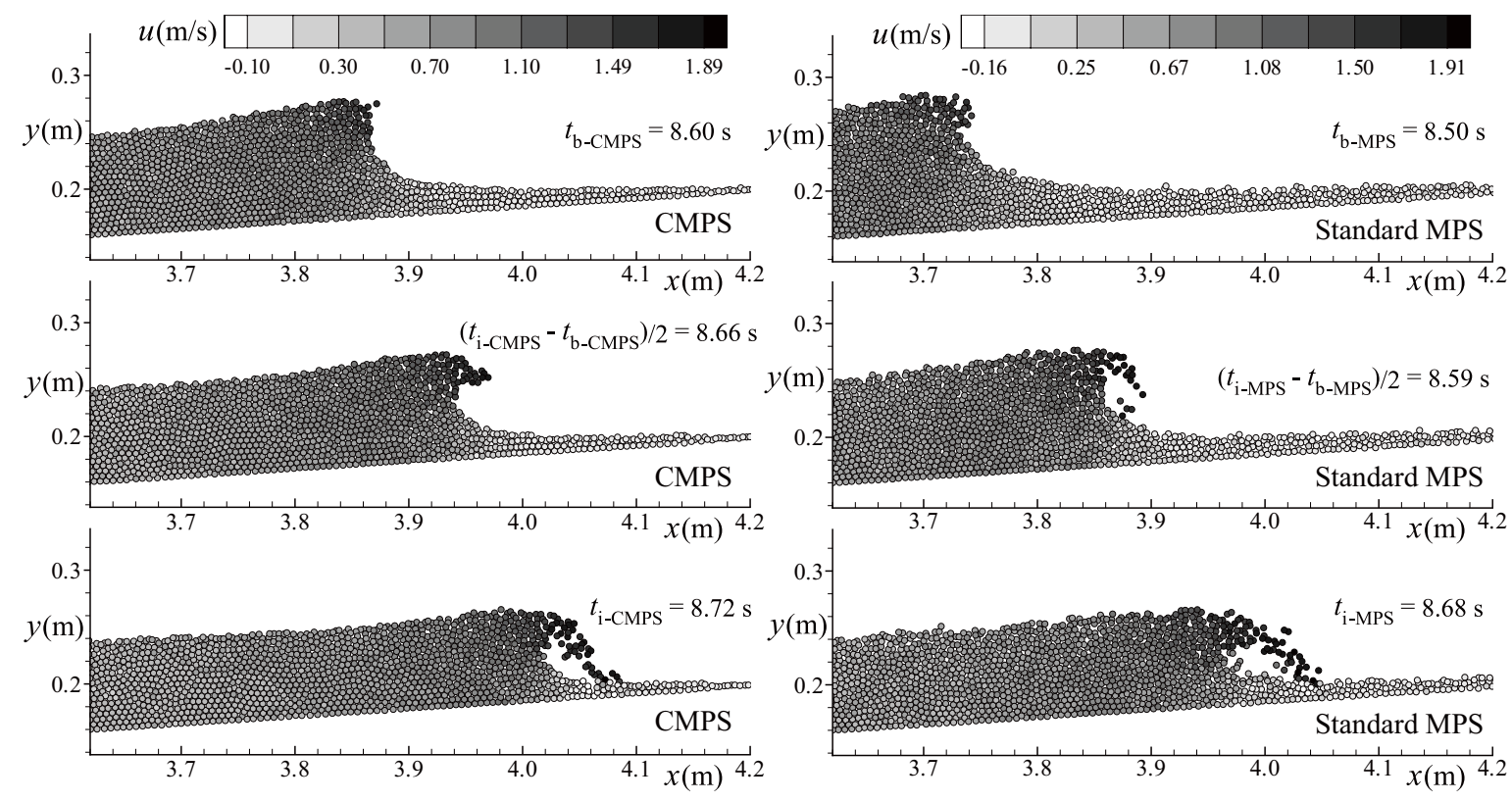

Fig. 16. CMPS (left) and standard MPS (right) snapshots of plunging wave breaking $\left(H_{0} / h_{0}=0.30\right)$ 


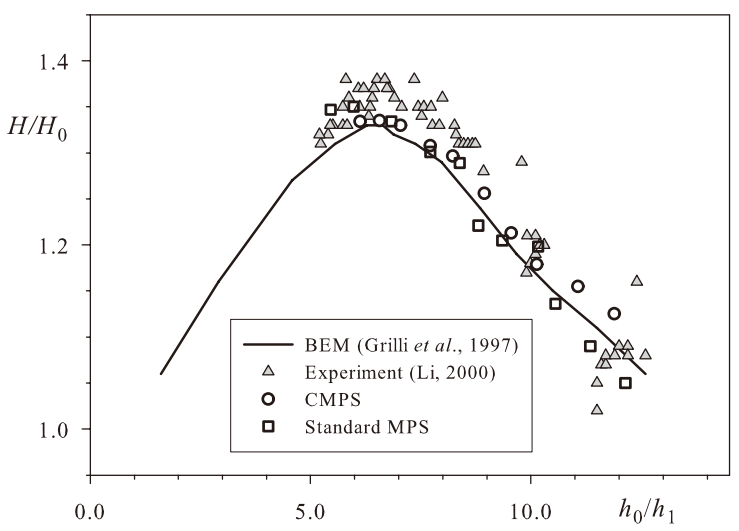

Fig. 17. Comparison of variation in wave height during breaking and post-breaking $\left(H_{0} / h_{0}=0.30\right)$

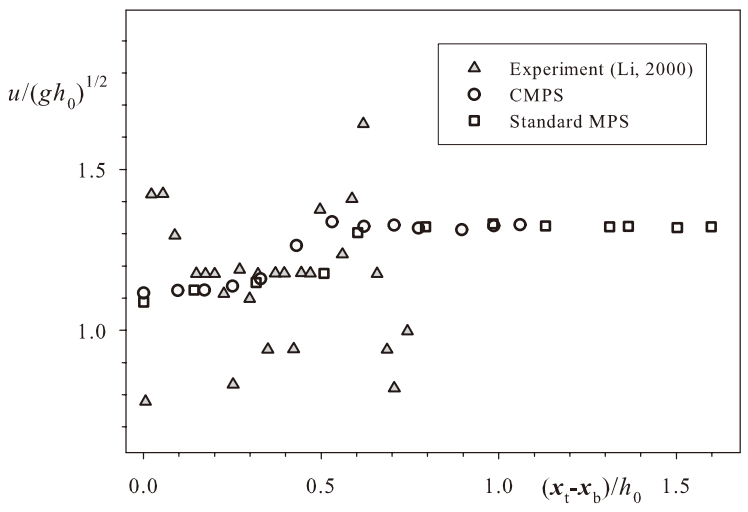

Fig. 18. Variation of the horizontal velocity of the plunging jet tip - comparison between the CMPS and standard MPS results $\left(H_{0} / h_{0}=0.30\right)$

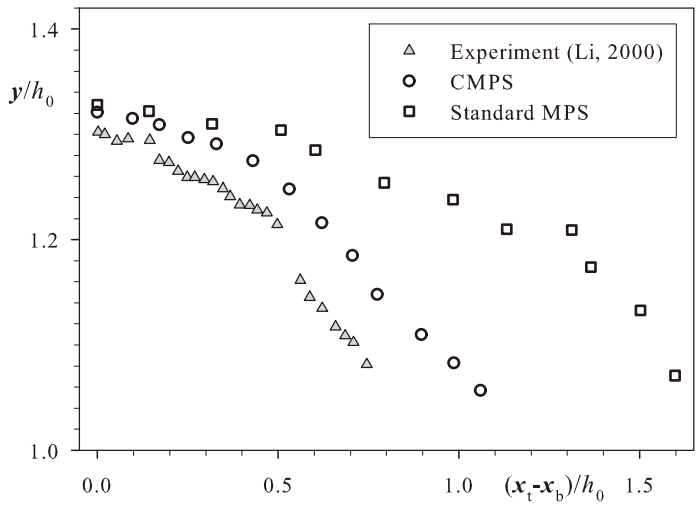

Fig. 19. CMPS and standard MPS predictions of the trajectory of the plunging jet tip $\left(H_{0} / h_{0}=0.30\right)$ 


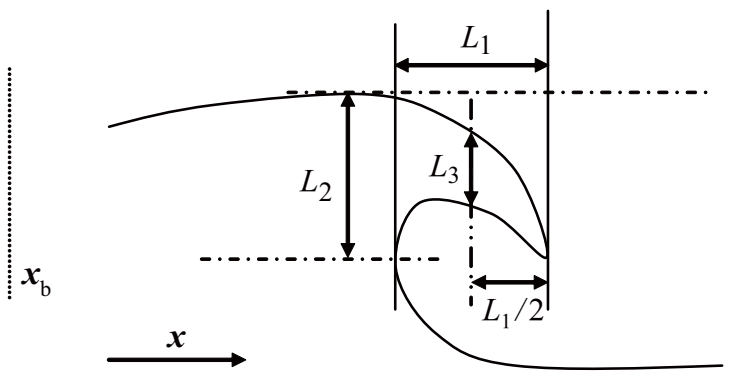

Fig. 20. Definition sketch of the geometrical parameters for describing the plunging jet
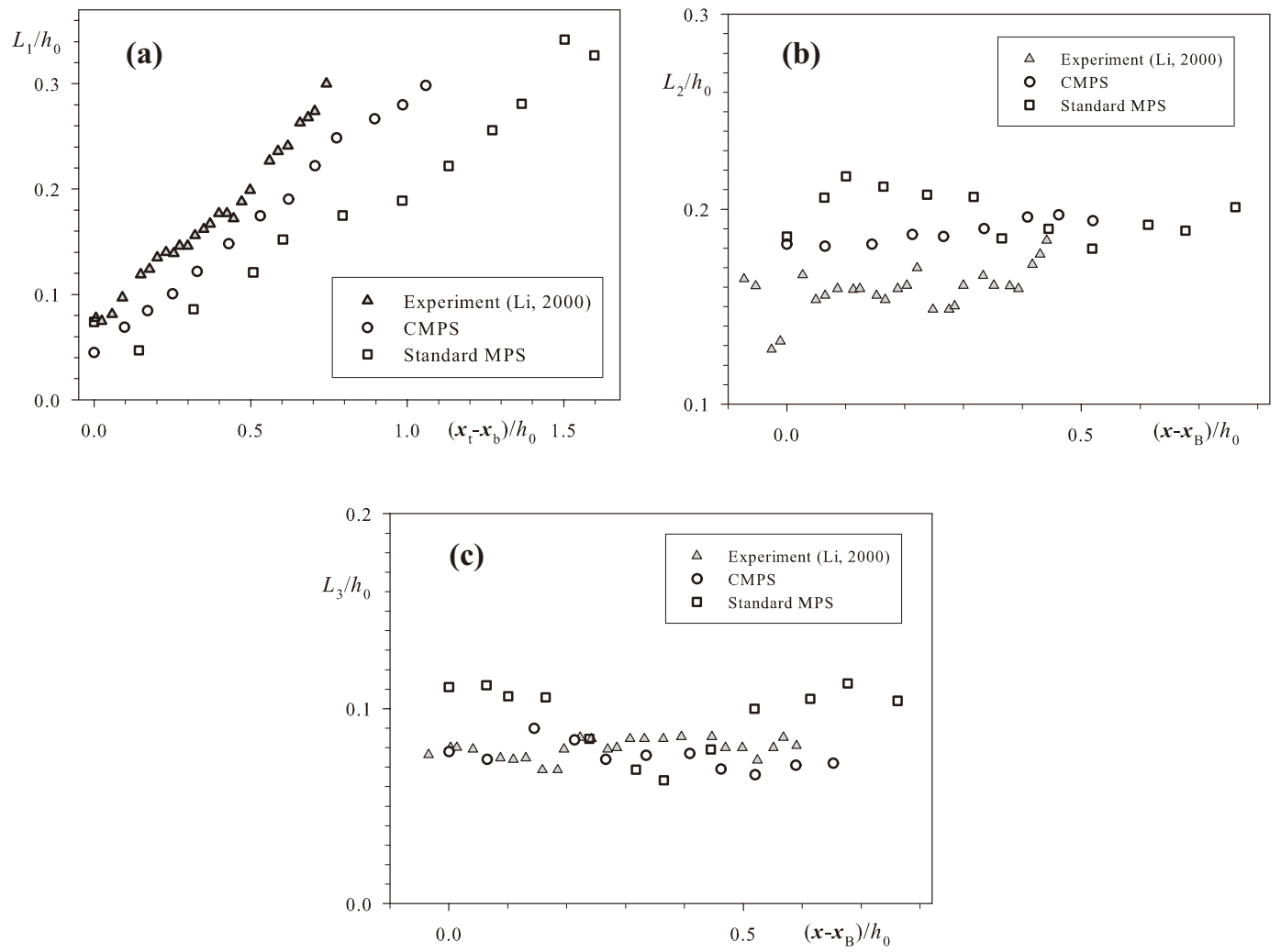

Fig. 21. Comparison between the CMPS and standard MPS results (a) Horizontal length $L_{1}$ (b) thickness $L_{2}$ (c) thickness $L_{3}$ of the plunging jet $\left(H_{0} / h_{0}=0.30\right)$ 


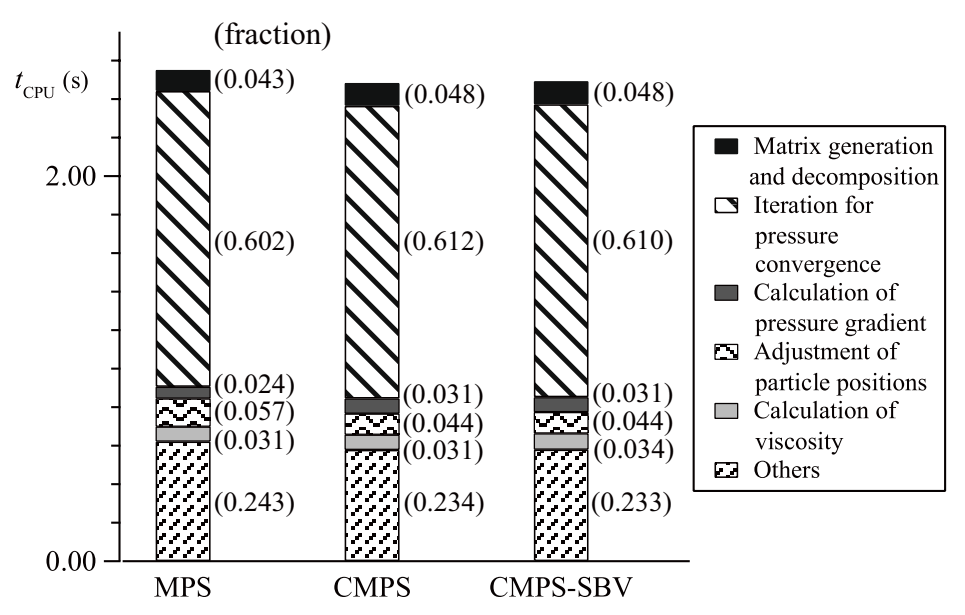

Fig. 22. CPU time of principal routines per time step in the MPS, CMPS and CMPS-SBV methods for calculation of wave breaking and post-breaking 